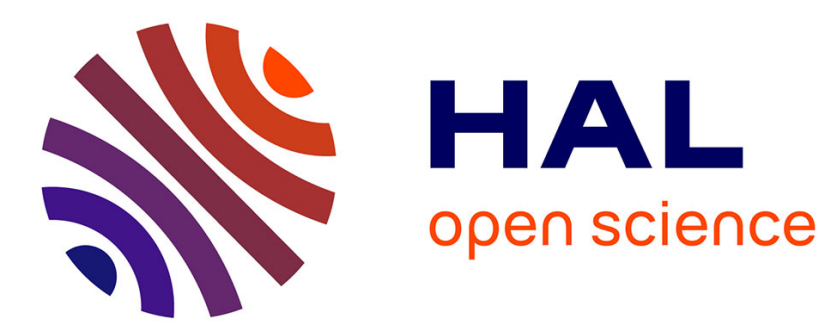

\title{
Dynamic Fragmentation of Brittle Solids: A Multi-Scale Model
}

\author{
Christophe Denoual, François Hild
}

\section{To cite this version:}

Christophe Denoual, François Hild. Dynamic Fragmentation of Brittle Solids: A Multi-Scale Model. European Journal of Mechanics - A/Solids, 2002, 21 (1), pp.105-120. 10.1016/S0997-7538(01)01187-

1. hal-00013965

\section{HAL Id: hal-00013965 \\ https://hal.science/hal-00013965}

Submitted on 16 Nov 2005

HAL is a multi-disciplinary open access archive for the deposit and dissemination of scientific research documents, whether they are published or not. The documents may come from teaching and research institutions in France or abroad, or from public or private research centers.
L'archive ouverte pluridisciplinaire HAL, est destinée au dépôt et à la diffusion de documents scientifiques de niveau recherche, publiés ou non, émanant des établissements d'enseignement et de recherche français ou étrangers, des laboratoires publics ou privés. 


\title{
Dynamic Fragmentation of Brittle Solids:
}

\author{
A Multi-Scale Model
}

\author{
Christophe Denoual and François HiLd
}

November 15, 2005

\begin{abstract}
Modeling dynamic fragmentation of brittle materials usually implies to choose between a discrete description of the number of fragments and a continuum approach of damage variables. A damage model that can be used in the whole range of loadings (from quasi-static to dynamic ones) is developed. The deterministic or probabilistic nature of fragmentation is discussed. Qualitative and quantitative validations are given by using a real-time visualization configuration for analyzing the degradation kinetics during impact and a moiré technique to measure the strains in a ceramic tile during impact. Finally, a closed-form solution of the change of the number of broken defects with the applied stress gives a way of optimizing the microstructure of ceramics for armor applications.
\end{abstract}

Keywords: Ceramic materials, probability and statistics, impact testing. 


\section{Introduction}

Bilayered armors with ceramics as front plate and steels as backing face have been used for several years to improve the efficiency of light or medium armors (den Reijer, 1991). The high hardness of ceramic materials favors projectile blunting and/or failure and spreads the kinetic energy on a large surface of the ductile backing. The weight of the armor is then reduced in comparison to an armor made of steel only. The response of a ceramic impacted by a steel rod is strongly dependent upon the impactor velocity. Low impact velocities (approximately less than $1000 \mathrm{~m} / \mathrm{s}$ ) lead to degradations such as cracking prior to a significant penetration. It follows that cracking is the prevalent mechanism to predict the residual properties of the ceramic before penetration and to assess its multi-hit capability. Higher impact velocities (ranging from about 1000 to $3000 \mathrm{~m} / \mathrm{s}$ ) usually lead to degradations in compressive and tensile modes (Espinosa et al., 1992).

Furthermore, the impactor can penetrate the ceramic layer even though some particular confinement conditions may prevent penetration (Bless et al., 1992; Hauver et al., 1994). Ultra-high velocities (greater than $3000 \mathrm{~m} / \mathrm{s}$ ) lead to a fully fragmented ceramic whose behavior is closer to that of a fluid rather than that of a solid material. Analytical models can be used to describe the response of the material (Tate, 1967; 1969).

The present paper is mostly concerned with the first impact regime where the main mechanism is fragmentation of brittle materials, and specifically ceramics used in light armors. During the first microseconds of impact, high stress waves are produced and lead to possible degradation in a compressive mode in the immediate surroundings of the projectile tip and in tensile mode in a widely extended zone. The fragmentation in tension, which extends over a larger zone than the degradation in a compressive mode, is one of the main mechanisms to identify (in terms of location, kinetics and anisotropic behavior due to cracking) for numerical simulations of impacts and penetration of projectiles. One can note that damage in compression involves very different mechanisms compared to damage in tension. 
For example, cracks propagating in mode II may lead to a different kinetics than that in mode I. Friction at the crack face has a significant influence on the damage description (Espinosa et al., 1992; Halm and Dragon, 1998). Finally, the population of flaws that lead to crack nucleation may be different in tension and in compression. To avoid an overlapping of mechanisms that would make the model validation delicate, only damage in tension is considered herein.

In Section 2, a damage model describing the tensile fragmentation is derived. After the presentation of a simplified description for the initial (i.e., undamaged) material, the fragmentation is analyzed as an extension of the brittle fracture regime observed in quasistatic loadings by considering random arrays of cracks. A multi-scale approach is proposed to model fragmentation with no constrains on the stress rate. In particular, the transition between single and multiple fragmentation is analyzed. It follows that the domain of validity of a continuum and local approach is obtained. A so-called "Edge-On-Impact" configuration is used in Section 3 and allows for the observation of damage patterns and strain fields during impact. Results of multi-scale simulations are discussed with respect to experimental data. Finally, a material optimization, which makes use of a closed-form solution of the change of the number of fragments with time or stress, is performed in Section 4 to assess the ballistic performance of four different $\mathrm{SiC}$ grades.

\section{A Model for the Fragmentation of Brittle Materials}

The present model is based on a reduced set of hypotheses for the microstructure description before and during the change of damage. Because microcracking (which leads to structural failure) is assumed to be caused by crack nucleation and growth, the first part of this study is devoted to modeling the microstructure of brittle materials prior to tensile degradation ( $\S 2.1)$. Cracks are supposed to emanate from defects and relax the stresses in their surroundings (§2.2). A complete description of crack nucleation and propagation finally leads to a damage description and kinetic law (see $\S 2.3$ and $§ 2.5$ ). The transition between single and multiple fragmentation is discussed in $§ 2.4$. 


\subsection{Microstructure of the Undamaged Material}

For brittle materials, the analysis of failure during quasi-static loadings can be used to define the relevant features of the microstructure in terms of flaw density and failure stress distribution. The nucleation of a crack in brittle materials subjected to quasi-static tension is due to (point) defects defined by a failure strength $\sigma_{\mathrm{f}}(\underline{x})$. When an equivalent stress $\sigma(\underline{x})$, e.g., maximum principal stress, is greater than $\sigma_{\mathrm{f}}(\underline{x})$, a crack emanating from the defect leads to the failure of the whole structure. The failure strength is a random function related to the defect distribution and location within the material. Therefore, the ultimate strength of a ceramic specimen is not deterministic and a failure probability $P_{\mathrm{F}}$ can be described by a Weibull law (1939)

$$
P_{\mathrm{F}}=1-\exp \left[-\lambda_{\mathrm{t}}\left(\sigma_{\mathrm{F}}\right) Z_{\mathrm{eff}}\right] \quad \text { with } \quad \lambda_{\mathrm{t}}\left(\sigma_{\mathrm{F}}\right)=\lambda_{0}\left(\frac{\sigma_{\mathrm{F}}}{\sigma_{0}}\right)^{m}
$$

where $\lambda_{\mathrm{t}}$ is the defect density, $m$ the Weibull modulus, $\sigma_{0}$ the reference stress relative to a reference density $\lambda_{0}, \sigma_{\mathrm{F}}$ the failure stress (i.e., the maximum equivalent stress in the considered domain $\Omega$ ) and $Z_{\text {eff }}$ the effective volume, surface or length (Davies, 1973). The constant $\lambda_{0} / \sigma_{0}^{m}$ is the so-called Weibull scale parameter. In the following, when no special mention is made, the development is valid for any space dimension $n$ (i.e., 1, 2 or 3 ). Otherwise, it will be clearly stated for which space dimension the results are valid. It can be noted that the previous formulation (i.e., Eqn. (1)) enters the framework of a Poisson point process of intensity $\lambda_{\mathrm{t}}$ (Gulino and Phoenix, 1991; Jeulin, 1991). The microstructure of the undamaged material is therefore approximated by defects of density $\lambda_{\mathrm{t}}$ with random locations.

Moreover, the mean failure stress $\sigma_{\mathrm{w}}$ and the corresponding standard deviation $\sigma_{\mathrm{sd}}$ are given by

$$
\sigma_{\mathrm{w}}=\frac{\sigma_{0}}{\left(Z_{\mathrm{eff}} \lambda_{0}\right)^{\frac{1}{m}}} \Gamma\left(1+\frac{1}{m}\right), \sigma_{\mathrm{sd}}^{2}=\frac{\sigma_{0}^{2}}{\left(Z_{\mathrm{eff}} \lambda_{0}\right)^{\frac{2}{m}}} \Gamma\left(1+\frac{2}{m}\right)-\sigma_{\mathrm{w}}^{2}
$$

where $\Gamma$ is the Euler function of the second kind. The relationships given in Eqn. (2) are used to estimate the defect density $\lambda_{t}[\sigma(t)]$ by using quasi-static tests even in the dynamic range (Denoual and Riou, 1995): up to stress rates of $10 \mathrm{MPa} \times \mu \mathrm{s}^{-1}$, the Weibull parameters of a 
silicon carbide ceramic are identical.

\subsection{Simplification of the Damaged Material}

In the bulk of an impacted ceramic, damage in tension is observed when the hoop stress induced by the radial motion is sufficiently large to generate fracture in mode I initiating on the microdefects already mentioned in Section 2.1. It will be assumed that the initial defect population leading to damage and failure is identical when the material is subjected to quasistatic and dynamic loading conditions (Denoual and Riou, 1995). This statement corresponds to the assumption that a single defect (e.g., a void, a microcrack) breaks at a stress level that is weakly dependent on the stress rate. However, the broken defect population is strongly dependent on the stress history. For very low stress rates, only the dominant defect of the whole population breaks (i.e., the weakest link of a structure) and the Weibull law described in the previous section applies. When dynamic loadings are considered, a part (increasing with the stress rate) of the initial defect population is broken. This point will be described in Sections 2.3 and 2.4.

Closed-form expressions for the effective properties of various crack patterns are proposed in the literature (see for example (Kachanov, 1994)). These solutions are obtained for quasi-static loadings and when crack interactions are considered, mostly periodic patterns are used. In the present study, the approximations needed for an analytical estimation of effective elastic properties of the cracked solid no longer hold. The crack velocity has the same order of magnitude as the Rayleigh wave speed (Kanninen and Popelar, 1985; Freund, 1990) and stress equilibrium during damage change is never achieved.

The considered crack pattern is made of penny-shaped cracks (instead of rectilinear cracks) of random locations. It follows that periodic homogenization techniques cannot be used to describe this type of dynamic cracking.

The link between microscopic and macroscopic scales is obtained by stating that the 
Gibbs' energy on a macroscopic scale is equal to the average specific enthalpy on a microscopic scale

$$
2 \rho \Phi=\underline{\underline{\underline{\Sigma}}}: \underline{\underline{\underline{S}}}^{\mathrm{D}}: \underline{\underline{\Sigma}}=\frac{1}{Z} \int_{\Omega} \underline{\underline{\sigma}}(\underline{x}): \underline{\underline{\underline{S}}}: \underline{\underline{\sigma}}(\underline{x}) \mathrm{d} \underline{x}
$$

where $\underline{\underline{\underline{S}}}^{\mathrm{D}}$ is the compliance tensor of the damaged material (and $\underline{\underline{\underline{S}}}$ is that of an undamaged material), $\rho$ the mass density, $\Omega$ a representative zone of measure $Z$, ':' the contraction with respect to two indices, $\underline{\underline{\sigma}}(\underline{x})$ the microscopic stress at point $\underline{x}$, and $\underline{\underline{\Sigma}}$ the macroscopic stress defined by

$$
\underline{\underline{\Sigma}}=\frac{1}{Z} \int_{\Omega} \underline{\underline{\sigma}}(\underline{x}) \mathrm{d} \underline{x} .
$$

The aim of this section is to introduce all the microscopic aspects of fracture to describe the microstructure of damaged material. A simplified stress field is proposed in the following section for a single crack and then extended to a random population of cracks.

\subsubsection{Single Crack}

Stress tensors are now expressed as vectors by using Voigt's notations and fourth order tensors are reduced to second order ones. When a fracture is initiated on a defect $k$ located at $\underline{x}_{k}$, the stress state around the propagating crack is a complex function of time, crack velocity and stress wave celerity. For a crack of normal $\underline{n}=\underline{x}_{1}$ submitted to a far field $\underline{\sigma}^{0}$, the microscopic stress field $\underline{\sigma}(\underline{x})$ at point $\underline{x}$ can be written as

$$
\underline{\sigma}(\underline{x})=[\underline{\underline{1}}-\underline{\underline{R}}(\underline{x})] \underline{\sigma}^{0}
$$

where $\underline{\underline{R}}(\underline{x})$ is a second order tensor accounting for stress modifications (mainly stress relaxation) around a crack.

In the appropriate coordinate system, the stress applied to the crack can be simplified as a uniform normal stress $\sigma_{\mathrm{n}}^{0}$ in addition to a uniform tangential loading $\sigma_{\mathrm{t}}^{0}$ (i.e., modes I and II). The quasi-static solution of stress relaxation around a penny-shaped crack given by Fabrikant (1990) is used as an approximation of the relaxed stress state during a dynamic loading. The relevant stress fields are plotted in Fig. 1. One can observe that for a crack 
normal aligned along direction $\underline{x}_{1}$, and a far field stress $\sigma_{\mathrm{n}}^{0}$, only the stress $\sigma_{11}(\underline{x})$ is relaxed over an important zone. For a pure tangential far field stress $\sigma_{\mathrm{t}}^{0}$ in the $\left(\underline{x}_{1}, \underline{x}_{2}\right)$ plane, only the component $\sigma_{12}(\underline{x})$ is significantly relaxed. Even though the whole relaxation tensor can be used, for the sake of simplicity, only the first and second most relaxed stress fields are considered in the following. Two relaxation components $R_{\mathrm{n}}$ and $R_{\mathrm{t}}$ are defined as

$$
\begin{aligned}
& \sigma_{11}(\underline{x})=\left[1-R_{\mathrm{n}}(\underline{x})\right] \sigma_{\mathrm{n}}^{0}, \\
& \sigma_{12}(\underline{x})=\left[1-R_{\mathrm{t}}(\underline{x})\right] \sigma_{\mathrm{t}}^{0} .
\end{aligned}
$$

A simplification of the stress field around a crack is proposed by using Boolean functions (Jeulin and Jeulin, 1981). An example of a Boolean representation of stress relaxation is given in Fig. 2-a and -b. Inside the Boolean functions $\Omega_{i j}\left(\underline{x}_{k}\right)$ associated to a defect of location $\underline{x}_{k}$, the stress state is supposed to be completely relaxed whereas outside the far field stress is applied

$$
\sigma_{i j}(\underline{x})= \begin{cases}0 & \text { if } \underline{x} \in \Omega_{i j}\left(\underline{x}_{k}\right), \\ \sigma_{i j}^{0} & \text { otherwise. }\end{cases}
$$

In the appropriate coordinate system, only two Boolean functions $\Omega_{\mathrm{n}}\left(\underline{x}_{k}\right)=\Omega_{11}\left(\underline{x}_{k}\right)$ and $\Omega_{\mathrm{t}}\left(\underline{x}_{k}\right)=\Omega_{12}\left(\underline{x}_{k}\right)$ are used to describe the stress relaxation for normal and tangential loadings, respectively.

It can be noted (see Eqns. (6), (7) and (8)) that the relaxation functions $R_{i j}(\underline{x})$ corresponding to the 'Boolean' stress field are also simplified (i.e., $R_{i j}(\underline{x})=1$ when $\underline{x} \in \Omega_{i j}\left(\underline{x}_{k}\right)$ and $R_{i j}(\underline{x})=0$ otherwise). The measure $Z_{\mathrm{n}}$ and $Z_{\mathrm{t}}$ of the relaxed (or obscured) zones $\Omega_{\mathrm{n}}\left(\underline{x}_{k}\right)$ and $\Omega_{\mathrm{t}}\left(\underline{x}_{k}\right)$ are estimated for each stress components by assuming that the definition of the macroscopic stress $\underline{\Sigma}$ (see Eqn. (4)) can be used for the real stress field and the 'Boolean' stress field defined in Eqn. (8)

$$
\begin{aligned}
\Sigma_{11} & =\frac{1}{Z} \int_{\Omega} \sigma_{11}(\underline{x}) \mathrm{d} \underline{x}=\left[1-\frac{1}{Z} \int_{\Omega} R_{\mathrm{n}}(\underline{x}) \mathrm{d} \underline{x}\right] \sigma_{\mathrm{n}}^{0} \\
& =\left(1-\frac{Z_{\mathrm{n}}}{Z}\right) \sigma_{\mathrm{n}}^{0}
\end{aligned}
$$


and similarly

$$
\Sigma_{12}=\left(1-\frac{Z_{\mathrm{t}}}{Z}\right) \sigma_{\mathrm{t}}^{0}
$$

where $Z$ is the measure of $\Omega$. A space scaling which modifies a length $l$ into its dimensionless counter-part $\bar{l}=l / a$ (where $a$ the radius of the considered crack) is used and allows one to derive a new expression for the measure of the obscured zones

$$
Z_{\mathrm{n}}=\int_{\Omega} R_{\mathrm{n}}(\underline{x}) \mathrm{d} \underline{x}=a^{n} \int_{\bar{\Omega}} R_{\mathrm{n}}(\underline{\bar{x}}) \mathrm{d} \underline{x}=a^{n} S_{\mathrm{n}}
$$

and

$$
Z_{\mathrm{t}}=\int_{\Omega} R_{\mathrm{t}}(\underline{x}) \mathrm{d} \underline{x}=a^{n} \int_{\bar{\Omega}} R_{\mathrm{t}}(\underline{\bar{x}}) \mathrm{d} \underline{x}=a^{n} S_{\mathrm{t}}
$$

where $\bar{\Omega}$ is a dimensionless zone, $S_{\mathrm{n}}$ and $S_{\mathrm{t}}$ are dimensionless shape parameters of the obscured zone for normal and tangential far field stresses, respectively, and $n$ is the space dimension ( $n=1$ for a line, $n=2$ for a shell, $n=3$ for a volume).

For sake of simplicity, it is assumed that cracks in brittle solids rapidly propagate at a constant velocity $k C_{0}\left(C_{0}=\sqrt{E / \rho}\right.$ where $E$ is the Young's modulus of the virgin material with $k$ a constant dependent on the material properties (Kanninen and Popelar, 1985) or (Freund, 1990)). Therefore, $Z_{\mathrm{n}}$ and $Z_{\mathrm{t}}$ become

$$
Z_{\mathrm{n}}=S_{\mathrm{n}}\left[k C_{0}(T-t)\right]^{n} \quad, \quad Z_{\mathrm{t}}=S_{\mathrm{t}}\left[k C_{0}(T-t)\right]^{n}
$$

where $T$ is the present time and $t$ the time to nucleation. It is worth mentioning that the shape parameters $S_{\mathrm{n}}$ and $S_{\mathrm{t}}$ may depend on the Poisson's ratio but they are independent of time, i.e., the relaxed zones are self-similar. A numerical estimation of $S_{\mathrm{n}}$ and $S_{\mathrm{t}}$ is carried out by using Fabrikant's solution and Eqns. (11) and (12). It follows that $S_{\mathrm{n}}=3.74$ and $S_{\mathrm{t}}=1.15$ for $\nu=0.15$ and $n=3$.

\subsubsection{Random Array of Cracks}

To model more complex situations (i.e., non-periodic penny-shaped cracks of various sizes, with random locations and propagating at high velocity), Boolean functions are used. Various 
non-periodic patterns can be obtained by simple operations such as dilution, superposition (see a review by Jeulin and Laurenge (1997)). For a random array of cracks during a dynamic loading, stress fields can be approximated by the unions $\Omega_{i j}^{\cup}$ of the whole set of Boolean functions $\Omega_{i j}\left(\underline{x}_{k}\right)$, each of them defined as the relaxed stress state around a single crack (Fig $2-\mathrm{c}$ and $-\mathrm{d}$ ) of random location $\underline{x}_{k}$

$$
\Omega_{i j}^{\cup}=\bigcup_{k} \Omega_{i j}\left(\underline{x}_{k}\right)
$$

The measure $Z_{i j}^{\cup}$ of $\Omega_{i j}^{\cup}$ is expressed as (Jeulin and Jeulin, 1981; Serra, 1982; Denoual et al., 1997a)

$$
\frac{Z_{i j}^{\cup}}{Z}=1-\exp \left\{-\lambda_{\mathrm{t}}[\sigma(t)] \mathcal{Z}_{i j}(t)\right\}
$$

where $\mathcal{Z}_{i j}(t)$ denotes the mean relaxed zone and $\lambda_{\mathrm{t}}[\sigma(t)]$ the intensity of the Poisson point process (Eqn. (1)). The mean relaxed zone $\mathcal{Z}_{i j}(t)$ is calculated by averaging at time $t$ the section of the obscured zones $Z_{i j}(t-\tau)$ for a nucleation at time $\tau$ and with a density $\frac{1}{\lambda_{t}[\sigma(t)]} \frac{\mathrm{d} \lambda_{\mathrm{t}}}{\mathrm{d} t}[\sigma(\tau)]$

$$
\mathcal{Z}_{i j}(t) \lambda_{\mathrm{t}}[\sigma(t)]=\int_{0}^{t} \frac{\mathrm{d} \lambda_{\mathrm{t}}}{\mathrm{d} t}[\sigma(\tau)] Z_{i j}(t-\tau) \mathrm{d} \tau
$$

where $Z_{11}(t)=Z_{\mathrm{n}}(t)$ and $Z_{12}(t)=Z_{\mathrm{t}}(t)$. A simple proof of these results is given in Appendix 1 .

The kinetics of damage is discussed in the following section. Both damage kinetics and description will be defined by using the simplified stress field description.

\subsection{Damage Kinetic Law}

The first approach dealing with stress relaxation has been proposed by Mott (1947) to model the fragmentation of a shell. Defects are assumed to be randomly located on a circular line. When the hoop stress increases, some of the defects break and relax the hoop stress. Because the following defects will break only in the non-relaxed (or non-obscured) zones, the increment of broken defects is equal to the increment of defects able to break multiplied by the fraction 
of loaded material. No analytical solutions were proposed but the same hypotheses were used in several models (see a review by Meyers (1994)). Another fragmentation model was proposed by Grady and Kipp (1980) and utilized for numerical calculations. The microstructure was also described through stress relaxation of spherical shape around penny-shaped cracks nucleated on initial defects.

To understand why a crack nucleates, one has to model the interaction of nucleated defects and other defects that would nucleate. The space location of the defects is represented in a simple abscissa of an $\mathrm{x}-\mathrm{y}$ graph where the $\mathrm{y}$-axis represents time (or stress) to failure of a given defect. In this graph, a shaded 'cone' represents the expansion of the obscuration zone with time due to nucleation and propagation of a crack. A section of a cone can be a volume, a surface or a length, depending on the space dimension $n$ (see Fig. 3-a). Inside this zone, the stress is decreasing and no new nucleation can occur. An approximation of this zone is given by $\Omega_{\mathrm{n}}^{\cup}\left(\right.$ and $\left.\Omega_{\mathrm{t}}^{\cup}\right)$ i.e., the zone where the stress normal to the crack is decreasing is assumed to be equal to the Boolean zone where the stress is relaxed. The defects located outside the shaded cones can nucleate and produce their own increasing relaxation zone (e.g., defects nos. 1, 2 and 3 of Fig. 3-a). Inside the cones, the defects that should have broken do not nucleate (e.g., defects nos. 4 and 5 of Fig. 3-a) since they are shielded (or obscured).

The total flaw density $\lambda_{\mathrm{t}}$ can be split into two parts: $\lambda_{\mathrm{b}}$ (the broken flaws) and $\lambda_{\text {obsc }}$ (the obscured flaws). Furthermore, the distribution of total flaws in a zone of measure $Z$ is assumed to be modeled by a Poisson point process of intensity $\lambda_{t}[\sigma(t)]$ in accordance with Section 2.1 (Eqn. (1)). New cracks will initiate only if the defect exists in the considered zone and if it does not belong to the relaxed zone $\Omega_{\mathrm{n}}^{\cup}$

$$
\frac{\mathrm{d} \lambda_{\mathrm{b}}}{\mathrm{d} t}[\sigma(t)]=\frac{\mathrm{d} \lambda_{\mathrm{t}}}{\mathrm{d} t}[\sigma(t)] \times\left[1-\frac{Z_{\mathrm{n}}^{\cup}(t)}{Z}\right]
$$

with $\lambda_{\mathrm{b}}(0)=\lambda_{\mathrm{t}}(0)=0$.

For a very high stress rate, most of the initial defects nucleate cracks before any significant change of the obscured zones, i.e., $\mathrm{d} \lambda_{\mathrm{b}} / \mathrm{d} t \approx \mathrm{d} \lambda_{\mathrm{t}} / \mathrm{d} t$ and $Z_{\mathrm{n}}^{\cup}(t) / Z \approx 0$. Conversely, when 
a very low stress rate is applied, the obscured zone occupies the whole volume $\left(Z_{\mathrm{n}}^{\cup}(t) / Z \approx 1\right)$ after the first crack nucleation, i.e., the nucleation is stopped after the failure of the weakest defect. The initial defect population described by $\lambda_{\mathrm{t}}$ is therefore used for both dynamic fragmentation and quasi-static failure but the number of nucleated defects varies with respect to the stress rate. Another way of obtaining Eqn. (17) is given in Appendix 1 by using the horizon of a defect. For a defect $\mathrm{P}$, the horizon is defined as a space-time zone in which a defect always obscures P (Fig. 3-b).

The fraction of relaxed zones $Z_{\mathrm{n}}^{\cup}(t) / Z$ is a good approximation for a damage variable $D$ defined in the framework of Continuum Damage Mechanics (Lemaitre, 1992), with $D=0$ for the virgin material and $D=1$ for the fully damaged one

$$
D=\frac{Z_{\mathrm{n}}^{\cup}}{Z}
$$

It is worth noting that the damage description is not necessarily isotropic even though it is characterized through a volume ratio. Since the relaxation zones are relative to a cracking direction, an anisotropic damage description is needed. The case of multiple superimposed crack patterns is studied in Section 2.5 where different variables $D_{i}$ are used for each direction $i$ of cracking. For any stress rate, the kinetics of $D_{i}$ is given in differential form (according to classical results of Continuum Damage Mechanics (Lemaitre, 1992), the change of $D_{i}$ is stopped when $\left.\mathrm{d} \sigma_{i} / \mathrm{d} t \leq 0\right)$

$$
\frac{\mathrm{d}^{n-1}}{\mathrm{~d} t^{n-1}}\left(\frac{1}{1-D_{i}} \frac{\mathrm{d} D_{i}}{\mathrm{~d} t}\right)=\widehat{\lambda_{\mathrm{t}}}\left[\sigma_{i}(t)\right] n ! S\left(k C_{0}\right)^{\mathrm{n}} \text { when } \frac{\mathrm{d} \sigma_{i}}{\mathrm{~d} t}>0 \text { and } \sigma_{i}>0
$$

where $\sigma_{i}$ is the eigen stress associated to penny-shaped cracks of normal $\underline{x}_{i}$ and $\widehat{\lambda_{\mathrm{t}}}$ is the density of defects effectively broken in the considered zone $(n=1,2$ or 3$)$. The density $\widehat{\lambda_{\mathrm{t}}}$ is therefore of probabilistic nature and may depend on a given realization (i.e., one can have 2, $0,1,5$, etc. defect(s) broken for different finite elements $\Omega_{\mathrm{FE}}$ of volume $V_{\mathrm{FE}}$ submitted to the same prescribed loading).

When an infinite volume is considered $\widehat{\lambda_{\mathrm{t}}}$ is equal to $\lambda_{\mathrm{t}}$. For a finite size $Z_{\mathrm{FE}}$ of a given finite element $\Omega_{\mathrm{FE}}$, the probabilistic density $\widehat{\lambda_{\mathrm{t}}}$ is approximated by the first defect able to 
break in addition with the density $\lambda_{\mathrm{t}}$ (see also (Benz and Asphaug, 1994)). The density $\widehat{\lambda_{\mathrm{t}}}$ is either equal to zero (no broken defect), or equal to or greater than $1 / Z_{\mathrm{FE}}$, i.e., at least one defect is broken in $\Omega_{\mathrm{FE}}$ (see Fig. 4)

$$
Z_{\mathrm{FE}} \widehat{\lambda_{\mathrm{t}}}\left[\sigma_{i}(t)\right]= \begin{cases}0 & \text { if } \sigma_{i}(t) \leq \sigma_{k}, \\ \max \left[Z_{\mathrm{FE}} \lambda_{0}\left(\frac{\sigma_{i}(t)}{\sigma_{0}}\right)^{m}, 1\right] & \text { otherwise. }\end{cases}
$$

The parameter $\sigma_{k}$ is the failure stress of the first defect $k$ able to break in $\Omega_{\mathrm{FE}}$. The failure stress is obtained by random selection of a failure probability $\left.P_{\mathrm{F}} \in\right] 0 ; 1\left[\right.$ with $Z=Z_{\mathrm{FE}}$ and is a function of the Weibull parameters $\left(m, \lambda_{0} / \sigma_{0}^{m}\right)$ and the mesh size $Z_{\mathrm{FE}}$ (see Eqns. (1), (2) and $(15))$.

\subsection{Continuum vs. Discrete Approaches}

When Continuum Damage Mechanics is used in numerical simulations, the medium is assumed to be continuum on the scale of a finite element in which numerous cracks are expected to nucleate. However, crack densities may strongly vary over the structure and the analysis of fragmentation through a continuum modeling may be delicate. As an alternative, discrete element modeling has been proposed (Camacho and Ortiz, 1996; Mastilovic and Krajcinovic, 1999) when the fragment size is greater than or equal to the size of a finite element. Espinosa et al. (1998) have developed a continuum/discrete multi-scale model in which the finer scale is discrete and allows for the derivation of a continuum description on a higher scale. In the present section, characteristic scales are introduced and enable one to choose between continuum or discrete approaches.

When dynamic (and proportional) loadings are considered with a constant stress rate $\mathrm{d} \sigma_{i} / \mathrm{d} t=\dot{\sigma}$, one can define a dimensionless flaw density $\left(\bar{\lambda}=\lambda / \lambda_{\mathrm{c}}\right)$, time $\left(\bar{t}=t / t_{\mathrm{c}}\right)$, space measure $\left(\bar{Z}=Z / Z_{\mathrm{c}}\right)$ and stress $\left(\bar{\sigma}_{i}=\sigma_{i} / \sigma_{\mathrm{c}}\right)$ from the condition (Denoual and Hild, 1998)

$$
\lambda_{\mathrm{c}} Z_{\mathrm{c}}=1 \text { with } \lambda_{\mathrm{c}}=\lambda_{\mathrm{t}}\left(\dot{\sigma} t_{\mathrm{c}}\right) \text { and } Z_{\mathrm{c}}=Z_{\mathrm{n}}\left(t_{\mathrm{c}}\right)
$$

where the subscript ' $c$ ' denotes characteristic quantities. A characteristic stress is defined by $\sigma_{\mathrm{c}}=\dot{\sigma} t_{\mathrm{c}}$. Equation (21) expresses the fact that the characteristic zone of measure $Z_{\mathrm{c}}$ contains 
on average one flaw that may break at the characteristic time $t_{c}$. By using Eqns. (1) and (21), the characteristic parameters are given by

$$
t_{\mathrm{c}}=\left[\frac{\sigma_{0}^{m}}{\lambda_{0} S\left(k C_{0}\right)^{n} \dot{\sigma}^{m}}\right]^{\frac{1}{m+n}}, Z_{\mathrm{c}}=\left[\frac{\left(\sigma_{0} k C_{0}\right)^{m} S^{m / n}}{\lambda_{0} \dot{\sigma}^{m}}\right]^{\frac{n}{m+n}}, \sigma_{\mathrm{c}}=\left[\frac{\sigma_{0}^{m} \dot{\sigma}^{n}}{\lambda_{0} S\left(k C_{0}\right)^{n}}\right]^{\frac{1}{m+n}} .
$$

This scaling is useful, in particular, when closed-form expressions can be given for the nucleated defect density, damage kinetics and ultimate strength (Denoual and Hild, 1998). By using Eqns. (1), (15), and (16) a closed-form solution can be derived for the differential equation (17) in the case of a constant stress rate $\dot{\sigma}$ (see Section 4). By using Eqns. (15), (16), (18) and by assuming that $\widehat{\lambda_{\mathrm{t}}}=\lambda_{\mathrm{t}}$, the change of any of the damage parameters $D_{i}$ is deterministic (Denoual and Hild, 2000)

$$
D_{i}=1-\exp \left[-\frac{m ! n ! \bar{\sigma}_{i}^{m+n}}{(m+n) !}\right] .
$$

The applied stress $\Sigma_{i}$ is related to the local (or effective) stress $\sigma_{i}$ by $\sigma_{i}=\Sigma_{i} /\left(1-D_{i}\right)$. The ultimate strength $\left(\mathrm{d} \Sigma_{i} / \mathrm{d} \sigma_{i}=0\right)$, denoted by $\Sigma_{\max }$, is therefore expressed as

$$
\frac{\Sigma_{\max }}{\sigma_{\mathrm{c}}}=\left[\frac{1}{e} \frac{(m+n-1) !}{m ! n !}\right]^{\frac{1}{m+n}} .
$$

These closed-form solutions for quasi-static (Eqn. (2)) and dynamic loadings (Eqn. (24)) can be validated by using Monte-Carlo simulations. In a cubic volume of $1.7 \mathrm{~mm}^{3}$, a set of flaws of density $\lambda_{t}[\sigma(t)]$ is randomly located. When the stress rate increases (with a constant stress rate $\dot{\sigma}$ ), obscuration zones following the process described in Section 2.2.1 are modeled. The macroscopic stress is obtained by averaging the microscopic stress in the non-relaxed zones. The behavior of this 'finite volume' is not deterministic and numerous calculations have to be performed when average values are sought (e.g., average macroscopic ultimate strength and standard deviation). Such calculations are shown in Fig. 5 where the macroscopic ultimate strength is plotted against the stress rate $\dot{\sigma}$. It can be noted that the results obtained with the multi-scale model (Eqn. (19)) are very comparable (in terms of mean and standard deviation) to those given by Monte-Carlo simulations (Denoual and Hild, 2000), with a CPU time divided by 3000 . For a stress rate within $\left[0,500 \mathrm{MPa} \times \mu \mathrm{s}^{-1}\right]$, the ultimate strength is not modified by the loading rate. Consequently, the quasi-static Weibull solution (Eqn. (2)) applies. When $\dot{\sigma}$ increases by approximately one order of magnitude, the ultimate 
tensile strength follows the 'dynamic' Weibull solution (24).

During the single / multiple fragmentation transition, the difference between the dashed lines (Eqns. (2) and (24)) and the Monte-Carlo simulations does not exceed 10\%. The standard deviation significantly decreases in the case of multiple fragmentation when the stress rate increases. Furthermore, for S-SiC ceramics, a stress rate up to $10 \mathrm{MPa} \times \mu \mathrm{s}^{-1}$ has shown no stress rate effect on the mean failure strength (Denoual and Riou, 1995). This observation is in good agreement with the result shown in Fig. 5.

The closed-form solutions for quasi-static (Eqn. (2)) or dynamic regimes (Eqn. (24)) are now used to determine when discrete or continuum approaches can be used. The transition between single and multiple fragmentation can be estimated as the intersection between the weakest link and the multiple fragmentation solutions (see Fig. 5)

$$
\Sigma_{\max }(\dot{\sigma})=\sigma_{\mathrm{w}}
$$

The transition defined by Eqn. (25) leads to the following inequalities

$$
\begin{cases}\dot{\sigma} Z^{\frac{m+n}{m n}}<f & \text { Single fragmentation } \\ \dot{\sigma} Z^{\frac{m+n}{m n}} \geq f & \text { Multiple fragmentation }\end{cases}
$$

with

$$
f=\sigma_{0} \lambda_{0}^{-1 / m} S_{n}^{1 / n} k C_{0}\left[e \frac{m ! n !}{(m+n-1) !} \Gamma\left(\frac{m+1}{m}\right)^{m+n}\right]^{\frac{1}{n}}
$$

This transition does not only depend upon material (Weibull) parameters but also involves the size $Z$ of the considered element and the applied stress rate $\dot{\sigma}$. The response of a large element can be considered as 'dynamic' for low stress rates although the same material follows the weakest link hypothesis for the same loading applied on a smaller element. There is therefore no intrinsic relationship between material parameters and characteristic scales to describe the fragmentation of brittle materials. It is a combination of material parameters, size and stress rate since there is a competition between local (increasing) stress rate and stress relaxation 
around cracks. By using the characteristic space measure $Z_{c}$, Eqn. (26) can be rewritten as

$$
\begin{cases}\frac{Z}{Z_{\mathrm{c}}(\dot{\sigma})}<g(m) & \text { Single fragmentation } \\ \frac{Z}{Z_{\mathrm{c}}(\dot{\sigma})} \geq g(m) & \text { Multiple fragmentation }\end{cases}
$$

with

$$
g(m)=\left[e \frac{m ! n !}{(m+n-1) !} \Gamma\left(\frac{m+1}{m}\right)^{m+n}\right]^{\frac{m}{m+n}} .
$$

The size $Z_{\mathrm{c}}$ can therefore be considered as the characteristic scale for which a single / multiple fragmentation transition is observed. Furthermore, Fig. 5 shows that, when $Z / Z_{\mathrm{c}} \geq 1$, the ultimate strength scatter is very small i.e., when the stress rate increases, the characteristic scale of the fragmented ceramic decreases and the stress estimated over $Z$ becomes a good approximation of the average stress.

Furthermore, a hypothesis of uniformity of the damage variables over the horizon (see Fig. 3) is needed in a local (and continuum) approach. When the mesh size $Z_{\mathrm{ZE}}$ is smaller than the horizon, two neighboring integration points have their horizons overlapping: a space location may be influenced by two a priori independent sets of variables. To avoid such a situation, the minimum mesh size must be greater than or equal to the horizon. Equation (23) shows that $D_{\mathrm{i}}\left(\bar{\sigma}_{\mathrm{i}}=1\right) \cong 0$ and $D_{\mathrm{i}}\left(\bar{\sigma}_{\mathrm{i}}=2\right) \cong 1$ (i.e., most of the damage change occurs during a time interval equal to $\left.t_{\mathrm{c}}\right)$. During $t_{\mathrm{c}}$, the measure of the horizon is limited by $Z_{\mathrm{n}}\left(t_{\mathrm{c}}\right)=Z_{\mathrm{c}}$. Therefore the minimum mesh size is $Z_{\mathrm{c}}$. The size $Z_{\mathrm{c}}$ is dependent on the loading rate: the higher the stress rate, the smaller the mesh size. This is consistent with the general practice of mesh refining when shock waves are suspected to occur. The characteristic size can be used in $\mathrm{FE}$ computations in which the mesh size $Z=Z_{\mathrm{FE}}$ has to be greater than or equal to $Z_{\mathrm{c}}$ to use a continuum (and deterministic) description of damage (Eqn. (28)).

The proposed scaling allows one to determine whether a continuum or discrete approach can be used. In the single fragmentation regime, a discrete (and non-local) method is a natural way of dealing with failure. Conversely, in the multiple fragmentation regime, the scatter 
in terms of overall behavior and failure strength becomes small. In that case, a classical Continuum (and local) Mechanics approach can be used. In the transition regime, discrete approaches may no longer be needed while Continuum (Damage) Mechanics hypotheses are not yet reasonable.

\subsection{Damage Description}

The aim of this section is to estimate of the compliance tensor $\underline{S}^{\mathrm{D}}$ of a damaged body. The case of cracks in one direction is analyzed in Section 2.5.1 and generalized thereafter to multiple crack patterns in Section 2.5.2.

\subsubsection{Cracks in One Direction}

The damage state can be described by using only one scalar variable $D_{1}$. Let the shape parameter $S_{\mathrm{t}}$ be written as a fraction $\alpha$ of $S_{\mathrm{n}}$

$$
S_{\mathrm{t}}=\alpha S_{\mathrm{n}}
$$

with $\alpha \approx 0.31$ for $\nu=0.15$ and $n=3$, see the numerical evaluation of $S_{\mathrm{t}}$ and $S_{\mathrm{n}}$ in Section 2.2.1.

It follows (see Eqns. (15), (16) and (18)) that

$$
1-\frac{Z_{\mathrm{t}}}{Z}=\left(1-D_{1}\right)^{\alpha}
$$

Consequently, there is a compliance increase for the 55 and 66 components of the compliance tensor

$$
\underline{\underline{S}}^{\mathrm{D}}=\frac{1}{E}\left[\begin{array}{cccccc}
\frac{1}{1-D_{1}} & -\nu & -\nu & 0 & 0 & 0 \\
-\nu & 1 & -\nu & 0 & 0 & 0 \\
-\nu & -\nu & 1 & 0 & 0 & 0 \\
0 & 0 & 0 & 1+\nu & 0 & 0 \\
0 & 0 & 0 & 0 & \frac{1+\nu}{\left(1-D_{1}\right)^{\alpha}} & 0 \\
0 & 0 & 0 & 0 & 0 & \frac{1+\nu}{\left(1-D_{1}\right)^{\alpha}}
\end{array}\right]_{\left(\underline{d}_{1}, \ldots .\right)}
$$


where $D_{1}$ is the damage variable due to cracks in the direction $\underline{d}_{1}$. It can be noted that a crack in direction $\underline{d}_{1}$ is surrounded by a zone $Z_{\mathrm{t}}$ that relaxes the shear stresses $\sigma_{12}(\underline{x})$ and $\sigma_{13}(\underline{x})$.

\subsubsection{Cracks in Multiple Directions}

When three orthogonal crack patterns are superimposed (i.e., the Boolean functions can be superimposed), the compliance tensor $\underline{\underline{S}}^{\mathrm{D}}$ is obtained by using Eqn. (3)

$$
\underline{\underline{S}}^{\mathrm{D}}=\frac{1}{E}\left[\begin{array}{cccccc}
\frac{1}{1-D_{1}} & -\nu & -\nu & 0 & 0 & 0 \\
-\nu & \frac{1}{1-D_{2}} & -\nu & 0 & 0 & 0 \\
-\nu & -\nu & \frac{1}{1-D_{3}} & 0 & 0 & 0 \\
0 & 0 & 0 & \frac{1+\nu}{\left(1-D_{2}\right)^{\alpha}\left(1-D_{3}\right)^{\alpha}} & 0 & 0 \\
0 & 0 & 0 & 0 & \frac{1+\nu}{\left(1-D_{3}\right)^{\alpha}\left(1-D_{1}\right)^{\alpha}} & 0 \\
0 & 0 & 0 & 0 & 0 & \frac{1+\nu}{\left(1-D_{1}\right)^{\alpha}\left(1-D_{2}\right)^{\alpha}}
\end{array}\right]_{\left(\underline{d}_{1}, \underline{d}_{2}, \underline{d}_{3}\right)}
$$

The compliance tensor $\underline{\underline{S}}^{\mathrm{D}}$ is defined in the directions of cracking $\left(\underline{d}_{1}, \underline{d}_{2}, \underline{d}_{3}\right)$. These directions associated to $D_{1}, D_{2}$ and $D_{3}$ may change at each time step until $D_{1}$ reaches a threshold value $D_{\text {th }}=0.01$ (the effect of the threshold value was found to be negligible in the simulations). Then, only the direction $\underline{d}_{1}$ is locked, the other directions follow the eigen directions of $\underline{\underline{\sigma}}$, with the constraint to be perpendicular to $\underline{d}_{1}$. When $D_{2}$ reaches the threshold value, the whole directions $\underline{d}_{i}$ are locked. It can be noted that the same type of result can be obtained by using mathematical arguments on a second order damage tensor. The only change is the value of the power $\alpha: \alpha=\frac{1}{2}$ (Cordebois and Sidoroff, 1982). Lastly, another description of the stress field (e.g., relaxation function $\underline{\underline{R}}(\underline{x})$ obtained by a numerical analysis instead of using Fabrikant's solution) would probably lead to yet another value of the constant $\alpha$.

The model can handle superpositions of crack patterns in up to three perpendicular directions. This is especially interesting when a complete fragmentation of the material is expected due to the stress waves reflecting on free surfaces. Such an experiment with a postmortem analysis (Denoual and Hild, 2000) also shows that the orientation of cracks does not 
change during impact, i.e., the fragments are created by the superposition of an array of straight cracks. The inability to deal with rapidly rotating principal directions of stress is however a limitation of the model.

\section{Comparison with Experiments on SiC Ceramics}

Once the elastic properties and the Weibull parameters are known, the model has no other parameters to tune. A special emphasis will be put on silicon carbide ceramics. Since silicon carbide ceramics can be obtained by different processing routes, the present study mainly focuses on two $\mathrm{SiC}$ grades whose properties are listed in Table I. The first grade, referred to as S-SiC, was provided by Céramique et Composites (France). The second grade (SiC-B) has been manufactured by CERCOM (USA). The $\mathrm{S}-\mathrm{SiC}$ ceramic is naturally sintered (sintering temperature: $\left.2000^{\circ} \mathrm{C}\right)$. The end product is an $\alpha-\mathrm{SiC}(6 \mathrm{H}$ hexagonal structure). The material is not fully dense. No secondary phase can be observed but $\mathrm{B}_{4} \mathrm{C}$ inclusions are present (Riou, 1996) because boron was added to enhance diffusion during sintering. Transgranular failure is the dominant mechanism. On the other hand, $\mathrm{SiC}-\mathrm{B}$ ceramics are obtained by pressure assisted densification. Aluminum is used to eliminate porosities (processing temperature: $2000^{\circ} \mathrm{C}$, pressure: $15 \mathrm{MPa}$ ). An alumina-rich secondary (glassy) phase is present (Forquin, 2000). Because of the lower strength of the secondary phase, the failure mode is predominantly intergranular.

Tensile cracking can be observed during impact by using Edge On Impact (EOI) configurations instead of a real configuration where the degradation is 'hidden' in the bulk of the ceramic. These configurations are developed by the Ernst-Mach-Institut (EMI) in Germany (Hornemann et al., 1984; Winkler et al., 1989; Straßburger and Senf, 1994) and more recently by the Centre Technique d'Arcueil (CTA) in France (Riou et al., 1996; 1998). It can be shown that the same damage mechanism (i.e., damage in tension) is observed in EOI and in real impact configurations (Denoual et al., 1996). 
For low impact velocities $(<500 \mathrm{~m} / \mathrm{s})$ no damage in compression occurs in SiC ceramics (Denoual et al., 1997b) and the EOI configuration can therefore be used to validate the damage kinetic laws for numerical simulations of the behavior of light armors.

Figure 6-a (top) shows a stress rate map $4 \mu$ s after impact with the corresponding damaged zone (bottom). When damage is generated, the stress rate is about $10^{3} \mathrm{MPa} / \mu \mathrm{s}$. One can see in Fig. 6 that this loading cannot be modeled accurately by using either continuum or discrete approaches, i.e., more than one defect breaks but the material cannot be considered as continuum in a FE cell. It follows that the multi-scale model is used. It can be noted that for each numerical simulation the set of random numbers is characterized by an integer called the 'seed' of the random generator (Press et al., 1992). A given probabilistic simulation is then defined by this integer and can always be reproduced by using the same 'seed'.

\subsection{Real Time Visualization}

Real time visualizations of damage have been performed with the $\mathrm{SiC}-\mathrm{B}$ grade by using the Edge-on Impact configuration developed by the EMI (Straßburger et al., 1994). The velocity of a single crack has been measured (Riou et al., 1998) and is about $4800 \mathrm{~m} / \mathrm{s}$. The value of the parameter $k$ is thus equal to 0.4. A remark can be drawn on the shape of the damaged zone with respect to the impact velocity. With an impact of high velocity, the damage is homogeneous in a circular zone in front of the projectile (see Fig. $7-\mathrm{b}$ and $-\mathrm{c}$ ). Below a critical value depending on the material properties, damage is localized in thinner and thinner corridors when the velocity decreases. Even though this localization leads to larger fragments, it has been demonstrated (Denoual and Hild, 1998) that the transition between corridors and circular shapes of damage is not related to the single/multiple fragmentation transition. A more detailed observation of the experimental result of Fig. 7-b shows that a corridor contains a high density of cracks, corresponding to a high local stress rate. 
The random stress to failure (Fig. 7-a) is calculated by using Eqns. (1) and (2) for a FE volume of $1 \mathrm{~mm}^{3}$. For high stress rates (i.e., in front of the projectile and in the Hertz-like cone crack), many defects nucleate in a FE cell. For a velocity of $185 \mathrm{~m} / \mathrm{s}$, failure of an element set, which can be compared to macroscopic cracks, can be observed in addition to the continuous degradation generated at the edge of the projectile (see Fig. 7-b). However, there are some difficulties in handling macroscopic cracks. The failure of a FE cell is not always followed by a crack generation and propagation, and when such a crack is created, there is a tendency to follow the direction of the FE mesh. The description of crack propagation may be improved by considering the failure of interfaces between finite elements instead of bulk failure (Camacho and Ortiz, 1996; Espinosa et al., 1998; Mastilovic and Krajcinovic, 1999).

\subsection{Moiré Technique}

A second EOI configuration provides quantitative strain measurements over a field of $32 \times$ $32 \mathrm{~mm}^{2}$ during impact. Details on the moiré photography set-up can be found in (BertinMourot et al., 1997). The advantage of the moiré measurement is that a quantitative rather than qualitative analysis can be performed between experiments and simulations. Figure 8-a is the fringe pattern approximately $2 \mu$ s after impact.

The comparison of numerical and experimental strains is given in Fig. 8-b. The strain diagram is plotted for a point $\mathrm{M}$ at a distance of $13 \mathrm{~mm}$ from the surface hit by the projectile (circular mark in Fig. 8-a). It can be noted that the radial strain reaches an important value (of the order of $0.8 \%$ ) before any significant change of the hoop strain. This is consistent with a cylindrical stress wave in which the tensile strain is induced by the radial motion of the material. The multi-scale model is used to give probabilistic numerical simulations instead of the deterministic simulations proposed with a continuum model. That is, numerous simulations have to be performed when the average behavior is analyzed. Five hundred realizations of the simulation presented in Section 3.2 are performed with the multi-scale 
model (a CPU time of 4 minutes per realization is needed on an HP 715 workstation with the finite element package PamShock (1998)). The average and standard deviation of the hoop and radial strains are plotted in Fig. 8. The multi-scale model yields good predictions of the strain levels. All the experimental measurements fall in the grey shaded zone, i.e., the experiment may be compared to one realization of the 500 numerical simulations. The use of an anisotropic model is necessary if one wants to accurately predict the strain levels. An elastic computation underestimates both radial and hoop strains. An isotropic damage model would have given even lower strain levels (Denoual et al., 1996).

\section{Towards Material Optimization}

A fine fragmentation of the ceramic leads to a localized strain around the projectile tip. The energy needed for the penetration into an armor is thus reduced in comparison to a coarse fragmentation where large fragments spread the strain within the volume and consume energy (see (Woodward et al., 1994) and Fig. 9). Therefore, an optimization criterion assumed to be relevant for armor is that an increase of fragment size (i.e., a decrease of broken defect density) leads to an increase of structural strength. A closed-form solution for $\lambda_{\mathrm{b}}(t)$ (Eqn. (17)) is used with a constant stress rate of $5 \mathrm{MPa} \times \mathrm{ns}^{-1}$ and a maximum tensile stress $\sigma_{\max }$ of $1 \mathrm{GPa}$

$$
\lambda_{\max }\left(\bar{\sigma}_{\max }\right)=\lambda_{\mathrm{c}} \frac{m}{m+n}\left(\frac{m ! n !}{(m+n) !}\right)^{\frac{m+n}{m}} \gamma\left[\frac{m}{m+n}, \frac{(m+n) ! \bar{\sigma}_{\max }^{m+n}}{m ! n !}\right]
$$

where $\gamma[p, x]=\int_{0}^{x} t^{p-1} \exp (-t) \mathrm{d} t$ is the incomplete gamma function. When the maximum tensile stress is reached, the kinetics of broken flaw density is stopped (see Fig. 10-a). The material parameters chosen to be optimized are the mean failure stress $\sigma_{\mathrm{w}}$ and the Weibull modulus $m$. For each couple $\left(\sigma_{\mathrm{w}}, m\right)$, the Weibull parameter $\lambda_{0} / \sigma_{0}^{m}$ is computed by using Eqn. (2). The results are shown in Fig. 10-b where the broken defect density is plotted as a function of $\sigma_{\mathrm{w}}$ and $m$. One can observe a significant influence of the Weibull modulus on the defect density: the higher $m$, the higher the broken flaw density (i.e., a poor ballistic performance). Moreover, it can be noted that an increase of the average failure stress would not improve the performance of the ceramic if the Weibull modulus increases too. 
The SiC-B and S-SiC ceramics shown in Fig. 10-b have different microstructures, the low porosity of SiC-B ceramics leading to a high average failure stress with a reduced scatter (i.e., a high Weibull modulus). However, the S-SiC grade has the coarser fragmentation leading to a better ballistic performance (Beylat and Cottenot, 1996). Finally, two other grades of silicon carbide called SiC-HIP (Riou, 1996) and SiC-150 (Leroy, 1999) are plotted in Fig. 10-b. The $\mathrm{SiC}-\mathrm{HIP}$ grade exhibits better ballistic performances than the $\mathrm{S}-\mathrm{SiC}$ grade (Beylat and Cottenot, 1996), as shown in Fig. 10-b. The SiC-150 grade, which has a porosity of $10-14 \%$, shows that a good material must have a low Weibull modulus, i.e., a large scatter of failure stresses and a low porosity content, i.e., a high average failure stress.

\section{Summary}

A fragmentation model based on a mechanism of nucleation of flaws and stress relaxation around propagating cracks is derived. By construction, this approach is non-local and the horizon of a defect constitutes the key ingredient. When a constant stress rate is applied, a closed-form solution for the number of nucleated defects is given. A damage kinetic law is derived from the fragmentation model. The analysis of stress relaxation around the propagating cracks leads naturally to an anisotropic description of damage. A differential equation is obtained for the kinetics of damage variables in order to be implemented into a FE code. The probabilistic nature of this model will help in understanding the non-deterministic behavior of structures made of brittle materials and submitted to a wide range of loadings (from quasistatic to dynamic ones). This model is able to describe a high density of cracks of random location. It is therefore well suited for describing degradations from the very early stages (i.e., nucleation of few cracks) up to the onset of crack coalescence.

Since all the parameters are determined by analytical analyses or identified through quasi-static (independent) tests, the model can be considered as fully predictive. The local- 
ization of damage in corridors that appears for materials with high Weibull moduli and high failure strength (e.g., the SiC-B grade) is well reproduced by the model. The strain history during impact is also predicted, in particular when the material seems to be intensively damaged (e.g., the $\mathrm{S}-\mathrm{SiC}$ grade).

The set of hypotheses shows that this model can only be used for damage in tension. Damage in compression should lead to a very different model even if the same kind of mechanisms (i.e., flaw nucleation, obscuration zones) are used. Moreover, when rapid rotating stresses are considered, the resulting damage is obtained through the superposition of orthogonal damage patterns instead of changing the direction of the crack propagation. This may be seen as a limitation of the model, as long as the differences between superposition of damage pattern and rotating cracks in terms of overall structural response is proven.

The transition zone for which the number of nucleated flaws is greater than but nevertheless close to one in a FE cell is well reproduced by the multi-scale model. The corresponding behavior, neither continuum (deterministic and local) nor discrete (probabilistic and nonlocal) is one of the major features of this model. Lastly, it is expected that these models are applicable to other brittle materials (such as rock, glass or concrete). Since the numbers of parameters to identify is very limited and can be carried out under quasi-static loading conditions, the model can be tested on a large class of brittle materials.

\section{Acknowledgements}

This work was funded by DGA-DRET-STRDT and supervised by Dr. C.E. Cottenot at CTA. The authors wish to thank Dr. E. Straßburger and Dr. H. Senf for providing the experimental data of Fig. 7. The authors wish also to thank A. Trameçon from ESI for his valuable help in implementing the model in PamShock. 


\section{$7 \quad$ References}

Benz W., Asphaug E., 1994. Impact Simulations with Fracture. I. Method and Tests. Icarus 107, 98-116.

Bless S.J., Ben-Yami M., Apgar L., Eylon D., 1992. Impenetrable Targets Struck by High Velocity Tungsten Long Rod. In: Proc. $2^{\text {nd }}$ Int. Conf. on Structures under Shock and Impact, Portsmouth (UK).

Bertin-Mourot T., Denoual C., Deshors G., Louvigné P.-F., Thomas T., 1997. High Speed Photography of Moiré Fringes: Application to Ceramics under Impact. J. Phys. IV coll. III-C3, 311-316.

Beylat L., Cottenot C.E., 1996. Post Mortem Microstructural Characterization of SiC Materials After Interaction with a Kinetic Energy Projectile. In: Proc. SUSI '96 symposium, $459-468$.

Camacho G.T., Ortiz M., 1996. Computational Modeling of Impact Damage in Brittle Materials. Int. J. Solids Struct. 33, 2899-2938.

Cho K., Katz N., Bar-On I., 1995. Strength and Fracture Toughness of Hot Pressed SiC Materials. Ceram. Sci. and Eng. Proc., 16.

Cordebois J.-P., Sidoroff F., 1982. Endommagement anisotrope en élasticité et plasticité. J. Méc. Th. Appl., special issue, 45-60.

Davies D.G.S., 1973. The Statistical Approach to Engineering Design in Ceramics. Proc. Brit. Ceram. Soc. 22, 429-452.

Denoual C., Riou P., 1995. Comportement à l'impact de céramiques techniques pour blindages légers. CREA, Report 95 R 005 (in French).

Denoual C., Cottenot C., Hild F., 1996. On the Identification of Damage During Impact of a Ceramic by a Hard Steel Projectile. In: Proc. $16^{\text {th }}$ Int. Symp. on Ballistics, ADPA, $541-550$.

Denoual C., Barbier G., Hild F., 1997a. A Probabilistic Approach for Fragmentation of Ceramics under Impact Loading. C. R. Acad. Sci. Paris Série IIb 325, 685-691. 
Denoual C., Cottenot C., Hild F., 1997b. Analysis of the Degradation Mechanisms in an Impacted Ceramic. In: Proc. Shock Compression of Condensed Matter, Schmidt S.C., Dandekar D.P. and Forbes J.W. (Edts.), AIP Press, New York (USA), 427-430.

Denoual C., Hild F., 1998. On the Characteristic Scales Involved in a Fragmentation Process. J. Phys. IV France 8, 119-126.

Denoual C., Hild F., 2000. A Damage Model for the Dynamic Fragmentation of Brittle Solids. Comp. Meth. Appl. Mech. Eng. 183, 247-258.

Espinosa H.D., Raiser G., Clifton R.J., Ortiz M., 1992. Experimental Observations and Numerical Modeling of Inelasticity in Dynamically Loaded Ceramics. J. Hard Mat. 3, $285-313$.

Espinosa H.D., Zavattieri P.D., Dwivedi S.K., 1998. A Finite Deformation Continuum/Discrete Model for the Description of Fragmentation and Damage in Brittle Materials. J. Mech. Phys. Solids 46, 1909-1942.

Fabrikant V.I., 1990. Complete Solution to Some Mixed Boundary Value Problems in Elasticity. Adv. Appl. Mech. 27, 153-223.

Forquin P., Denoual C., Cottenot C.E., Rota L., Hild F., (2000). Experimental Approach and Modeling of the Compressive Behaviour of Two SiC Grades. J. Phys. IV 10, Pr9-735-740.

Freund L.B., 1990. Dynamic Fracture Mechanics, Cambridge University Press, Cambridge $(\mathrm{UK})$.

Grady D.E., Kipp M.E., 1980. Continuum Modeling of Explosive Fracture in Oil Shale. Int. J. Rock Min. Sci. Geomech. 17, 147-157.

Gulino R., Phoenix S.L., 1991. Weibull Strength Statistics for Graphite Fibres Measured from the Break Progression in a Model Graphite/Glass/Epoxy microcomposite. J. Mat. Sci. 26, 3107-3118.

Halm D., Dragon A., 1998. An Anisotropic Model of Damage and Frictional Sliding for Brittle Materials. Eur. J. Mech. A/Solids 17, 439-460.

Hauver G., Netherwood P., Benck R., Kecskes L., 1993. Ballistic Performance of Ce- 
ramic Targets. Army Symposium on Solids Mechanics, Plymouth (USA).

Hornemann U., Kalthoff J.F., Rothenhäusler H., Senf H., Winkler S., 1984. Experimental Investigation of Wave and Fracture Propagation in Glass - Slabs Loaded by Steel Cylinders at High Impact Velocities. EMI report E 4/84, Weil am Rhein (Germany).

Jeulin D., 1991. Modèles morphologiques de structures aléatoires et de changement d'échelle, Thèse d'État, University of Caen (in French).

Jeulin D., Jeulin P., 1981. Synthesis of Rough Surfaces by Random Morphological Models. Stereol. Iugosl. 3/Suppl. 1, 239-246.

Jeulin D., Laurenge P., 1997. Simulation of Rough Surfaces by Morphological Random Functions. J. Electronic Im. 6, 16-30.

Kachanov M., 1994. Elastic Solids with Many Cracks and Related Problems. Adv. Appl. Mech. 30, 259-445.

Kanninen M.F., Popelar C.H., 1985. Advanced Fracture Mechanics, Oxford University Press, New York (USA).

Lemaitre J., 1992. A Course on Damage Mechanics, Springer-Verlag, Berlin (Germany). Leroy Y., 1999. Private communication.

Mastilovic S., Krajcinovic D., 1999. High-Velocity Expansion of a Cavity within a Brittle Material, J. Mech. Phys. Solids 47, 577-600.

Meyers M.A., 1994. Dynamic Behavior of Materials, Wiley Interscience, New York (USA).

Mott N.F., 1947. Fragmentation of Shell Cases. Proc. Roy. Soc. London A189, 300-308.

Palika R., 1995. Private communication.

PamShock, 1998. User's manual, Pam System International, ESI.

Press W.H., Teukolsky S.A., Vetterling W.T., Flannery B.P., 1992. Numerical Recipes in C, Cambridge University Press, Cambridge (UK).

den Reijer P.C., 1991. Impact on Ceramic Faced Armor, PhD thesis, Delft Technical University.

Riou P., 1996. Contribution à l'étude de l'endommagement du carbure de silicium 
lors d'un impact de basse énergie : application aux blindages, PhD thesis, École Nationale Supérieure des Mines de Paris (in French).

Riou P., Denoual C., Cottenot C.E., 1998. Visualization of the Damage Evolution in Impacted Silicon Carbide Ceramics. Int. J. Impact Eng. 21, 225-235.

Serra J., 1982. Image Analysis and Mathematical Morphology, Academic Press, London $(\mathrm{UK})$.

Straßburger E., Senf H., 1994. Experimental Investigations of Wave and Fracture Phenomena in Impacted Ceramics. EMI report 3/94, Weil am Rhein (Germany).

Tate A., 1967. A Theory for the Deceleration of Long Rods after Impact. J. Mech. Phys. Solids 15, 387-399.

Tate A., 1969. Further Results in the Theory of Long Rods Penetration. J. Mech. Phys. Solids 17, 141-150.

Weibull W., 1939. A Statistical Theory of the Strength of Materials. Roy. Swed. Inst. Eng. Res. 151.

Winkler S., Senf H., Rothenhäusler, 1989. Wave and Fracture Phenomena in Impacted Ceramics. EMI report V 5/89, Weil am Rhein (Germany).

Woodward R.L., Gooch, Jr W.A., O’Donnell R.G., Perciballi W.J., Baxter B.J., Pattie S.D., 1994. A Study of Fragmentation in the Ballistic Impact of Ceramics. Int. J. Impact Eng. 15, 605-618. 


\section{Appendix 1}

The kinetics of nucleated flaw density or damage variable can be obtained by using the conditions of non-relaxation for a given defect by examining the inverse problem (Denoual et al., 1997). It consists in considering the past history of a defect that would break at a time $T$. The defect will break if no defects exist in its horizon. For a given defect D, its horizon is defined as a space-time zone in which a defect will always obscure P (Fig. 3). Outside the horizon a defect will never obscure P. Equation (17) becomes

$$
\frac{\mathrm{d} \lambda_{\mathrm{b}}}{\mathrm{d} t}(T)=\frac{\mathrm{d} \lambda_{\mathrm{t}}}{\mathrm{d} t}(T)\left[1-P_{\mathrm{o}}(T)\right] \quad \text { with } \quad \lambda_{\mathrm{b}}(0)=0 \quad \text { and } \quad \lambda_{\mathrm{t}}(0)=0
$$

where $1-P_{\mathrm{o}}$ is the probability that no defect exist in the horizon $\left(P_{\mathrm{o}}=\frac{Z_{\mathrm{n}}}{Z}\right)$. The variable $P_{\mathrm{o}}$ can be split into an infinity of events defined by the probability $\Delta P(t)$ of finding at $t$ a new defect during a time step $\mathrm{d} t$ in a zone $Z_{\mathrm{n}}(T-t)$. This probability increment is written by using a Poisson point process of intensity $\mathrm{d} \lambda_{\mathrm{t}} / \mathrm{d} t$. Those independent events can be used to derive the following expression for $P_{\mathrm{o}}$

$$
\begin{aligned}
1-P_{\mathrm{o}}(T) & =\Pi_{t}^{T}(1-\Delta P(t)) \\
& =\Pi_{t}^{T} \exp \left[-\frac{\mathrm{d} \lambda_{\mathrm{t}}}{\mathrm{d} t}(t) \Delta T Z_{\mathrm{n}}(T-t)\right] \\
& \approx \exp \left[-\int_{0}^{T} \frac{\mathrm{d} \lambda_{\mathrm{t}}}{\mathrm{d} t}(t) Z_{\mathrm{n}}(T-t) \mathrm{d} t\right]
\end{aligned}
$$

where $Z_{\mathrm{n}}(T-t)$ is the measure of the interaction zone at $t$ for a defect that would break at $T$. This completes the proof. 


\section{List of Tables}

I. Material properties of four $\mathrm{SiC}$ ceramics. 
Table I. Material properties of four SiC ceramics.

\begin{tabular}{lcccc}
\hline Parameters & S-SiC & SiC-B & SIC-HIP & SiC-150 \\
\hline Young's modulus $E(\mathrm{GPa})$ & 410 & 455 & 465 & 350 \\
Poisson's ratio $\nu$ & 0.15 & 0.16 & 0.15 & 0.25 \\
Density & 3.15 & 3.20 & 3.18 & $2.76-2.89$ \\
Porosity & $1.8 \%$ & 0 & $\mathrm{NA}$ & $10-14 \%$ \\
Weibull modulus $m$ & 9.3 & 27 & 8.6 & 15 \\
Mean failure strength $\sigma_{\mathrm{w}}(\mathrm{MPa})$ & 370 & 560 & 590 & 225 \\
Effective volume $Z_{\text {eff }}\left(\mathrm{mm}^{3}\right)$ & 1.7 & 1.5 & 1.2 & 1.4 \\
Number of samples & 65 & 30 & 26 & NA \\
Type of flexural test & $3-$ point & 4 -point & 3 -point & 3 -point \\
\hline
\end{tabular}

\#: (Denoual and Riou, 1995)

b: (Palika, 1995; Cho et al., 1994)

Ł. (Leroy, 1999) 


\section{List of Figures}

1 Example of stress relaxation functions for normal and tangential loadings. The normal stress $\sigma_{11}$ and shear stress $\sigma_{12}$ have the greatest relaxation zones for normal and tangential loadings, respectively. . . . . . . . . . . . . . 33

2 Example of simplification for a complex microstructure containing penny-shaped cracks submitted to a normal far field stress. The stress field around a single crack (a) is transformed into a Boolean function (b) on which Boolean operations (i.e., union) can be performed (d). This simplification is used as a representation of complex microstructures (c) for which effective elastic properties are delicate to obtain. For the sake of simplicity, only the stress field $\sigma_{11}$ is represented even if the method is applied on tensor fields. . . . . . . . . . . 34

3 a-Depiction of obscuration phenomena.

b-Horizon for a defect $P \ldots \ldots \ldots \ldots \ldots \ldots$

4 Change of broken flaw density with time predicted by the multi-scale model (solid line) and the fragmentation theory (dashed line) . . . . . . . . . . 36

5 Ultimate macroscopic strength vs. stress rate predicted by the multi-scale model and Monte-Carlo simulations (500 realizations for each points (Denoual and Hild, 2000)) for an S-SiC ceramic. . . . . . . . . . . . . 37

6 Requirements to be fulfilled to use a continuum damage model can be checked by using a maximum stress rate map.

a- Example of maximum stress rate for the first eigen stress $4 \mu s$ after impact (top) and the corresponding damaged zone (bottom). The line density is a function of broken flaws density and the line direction is that of cracking. $\mathrm{b}-$ The stress rate associated to locations where damage is generated leads to a complex behavior, neither discrete nor continuum. . . . . . . . . . . . . 38 
$7 \quad$ Numerical simulations of a SiC-B ceramic in an EOI configuration $(50 \times 100 \times 10$ elements of $\left.1 \times 1 \times 1 \mathrm{~mm}^{3}\right)$.

a- Example of random failure stress $\sigma_{k}$ for the first defect able to break in each FE cell.

b- and c- Tile upper part: simulations (multi-scale model), tile lower part: experiments. Damaged zones $\left(D_{1}>0.5\right.$ in the dark zones) for two impact velocities. . . . . . . . . . . . . . . . . . . . . . . 39

8 a- Typical example of moiré fringes.

b- Strain change given by a moiré technique (dots) and by the multi-scale model (plain curve: average, grey bandwidth: \pm standard deviation). . . . . . 40

$9 \quad$ Strains around a penetrating projectile when the fragmentation is coarse (left) and fine $($ right $) \ldots \ldots \ldots \ldots \ldots \ldots$. . . . . . . . . . . . . . . . . .

10 a- Stress history for material optimization. The dashed curve is any positive monotonically decreasing function.

b- Broken flaw density map as function of Weibull modulus $m$ and average failure stress $\sigma_{\mathrm{w}}$. The four grades of silicon carbide are depicted by crosses. . . 42 


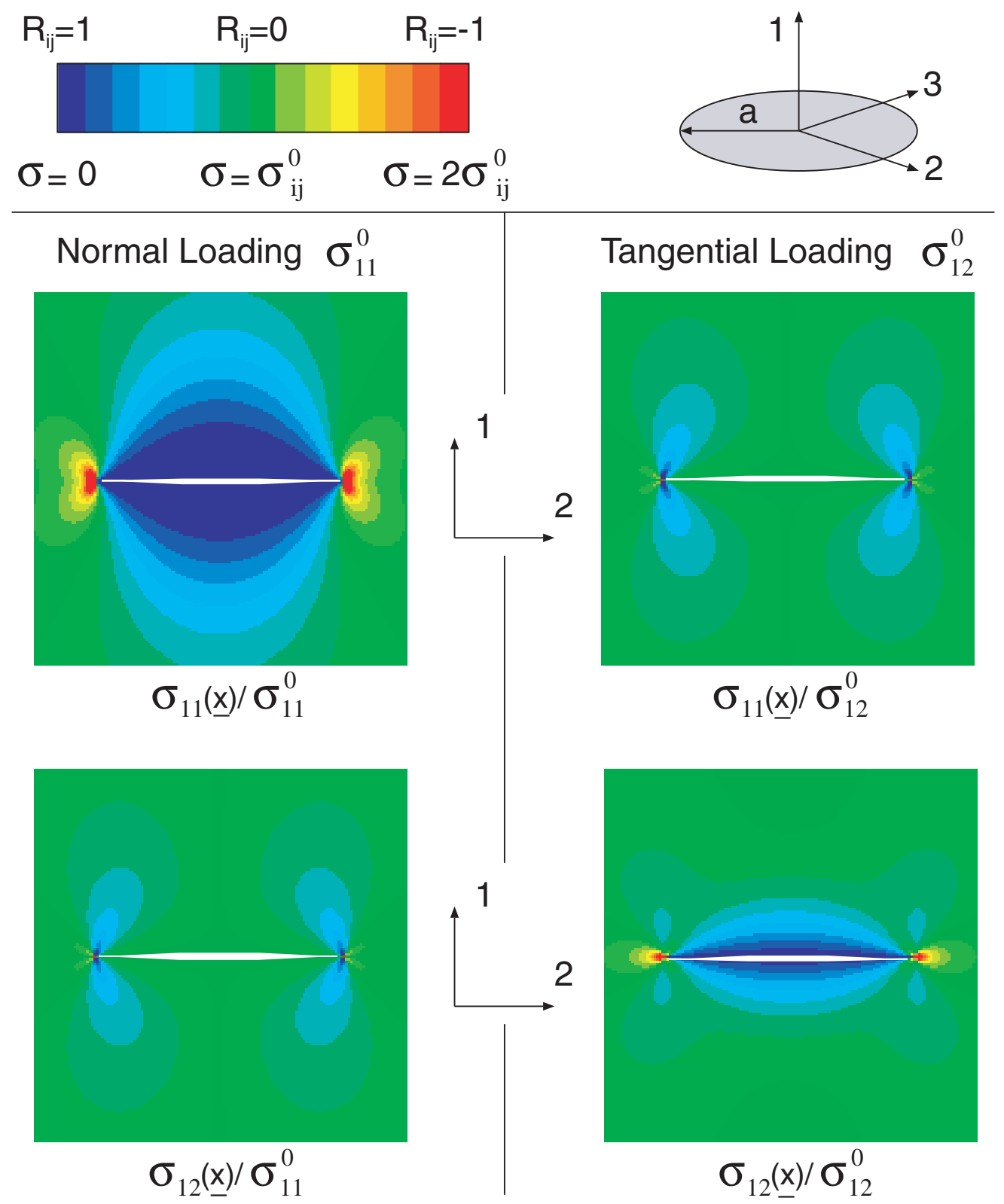

Figure 1: Denoual and Hild

Figure 1: Example of stress relaxation functions for normal and tangential loadings. The normal stress $\sigma_{11}$ and shear stress $\sigma_{12}$ have the greatest relaxation zones for normal and tangential loadings, respectively. 


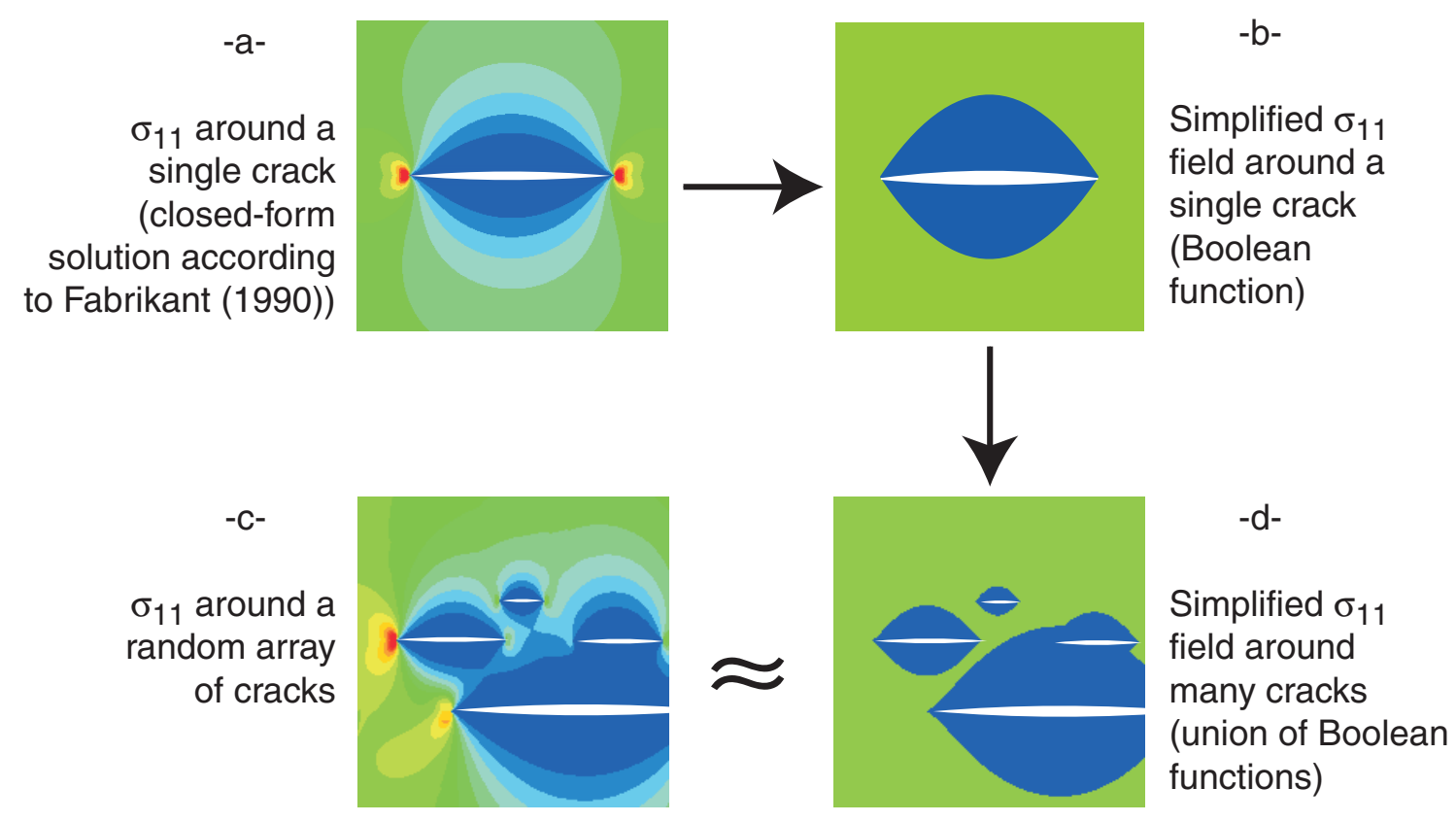

Figure 2: Denoual and Hild

Figure 2: Example of simplification for a complex microstructure containing penny-shaped cracks submitted to a normal far field stress. The stress field around a single crack (a) is transformed into a Boolean function (b) on which Boolean operations (i.e., union) can be performed (d). This simplification is used as a representation of complex microstructures (c) for which effective elastic properties are delicate to obtain. For the sake of simplicity, only the stress field $\sigma_{11}$ is represented even if the method is applied on tensor fields. 


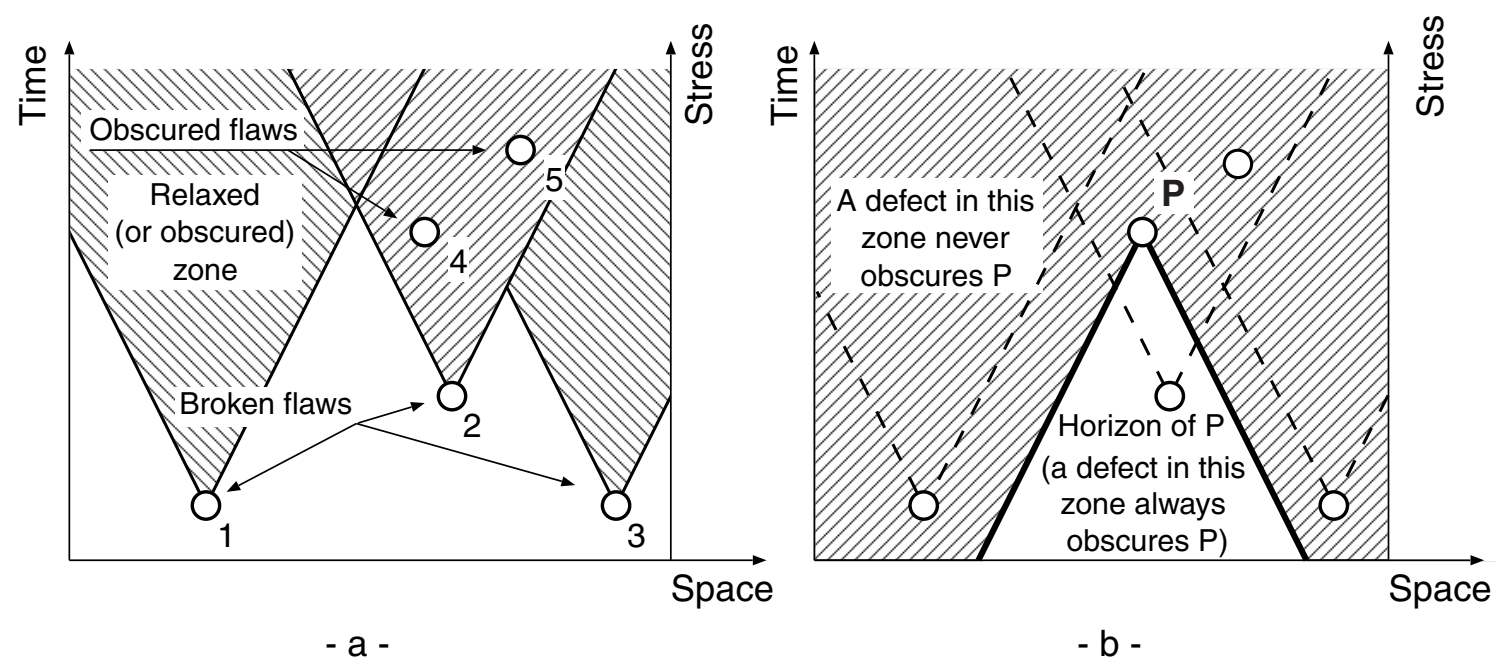

Figure 3: Denoual and Hild

Figure 3: a-Depiction of obscuration phenomena.b-Horizon for a defect $P$. 


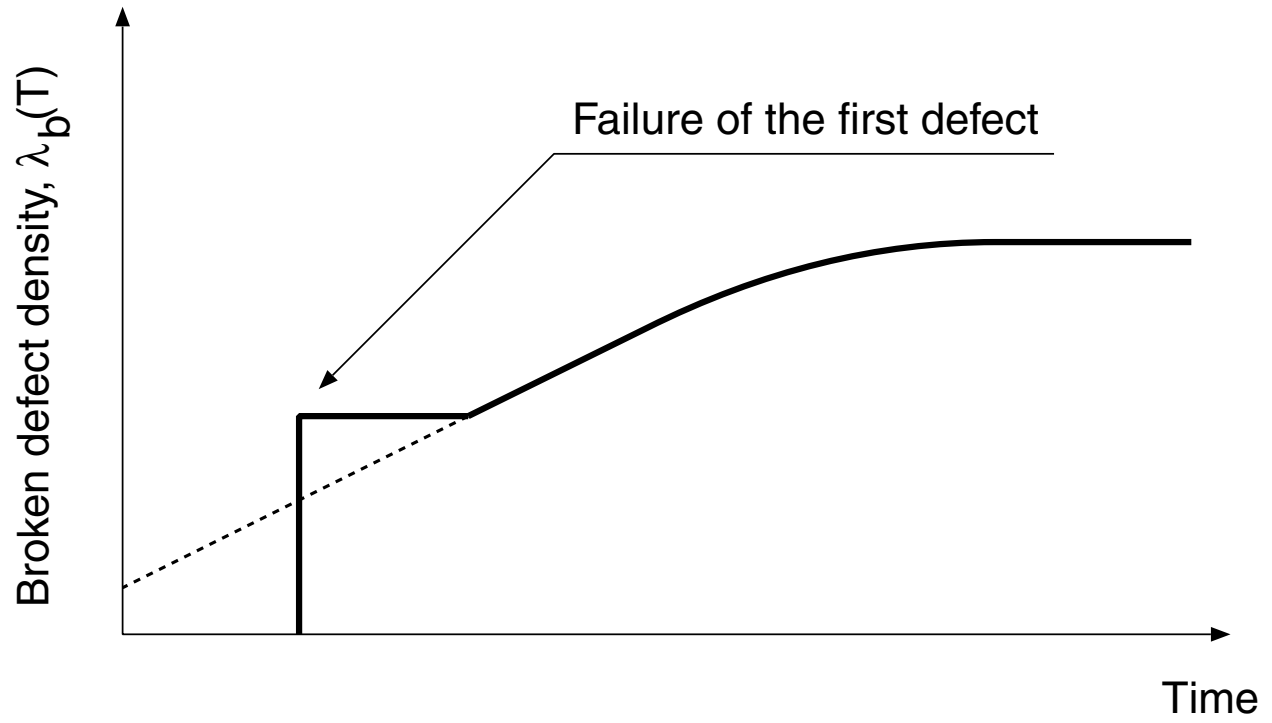

Figure 4: Denoual and Hild

Figure 4: Change of broken flaw density with time predicted by the multi-scale model (solid line) and the fragmentation theory (dashed line). 


\section{---- Weibull law Monte-Carlo simulations}

Dimensionless volume, $\mathrm{Z} / \mathrm{Z}_{\mathrm{C}}$

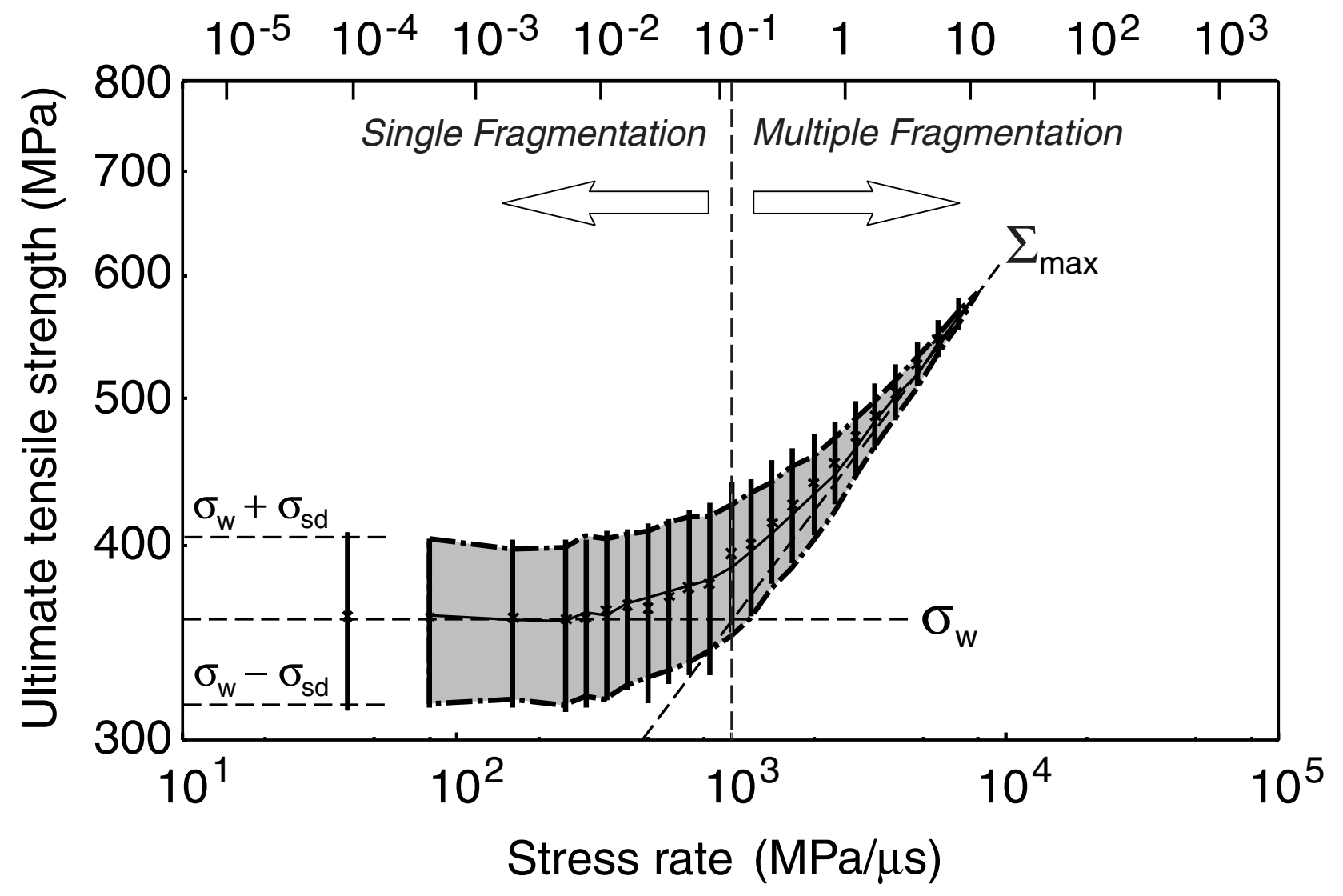

Figure 5: Denoual and Hild

Figure 5: Ultimate macroscopic strength vs. stress rate predicted by the multi-scale model and Monte-Carlo simulations (500 realizations for each points (Denoual and Hild, 2000)) for an $\mathrm{S}-\mathrm{SiC}$ ceramic. 


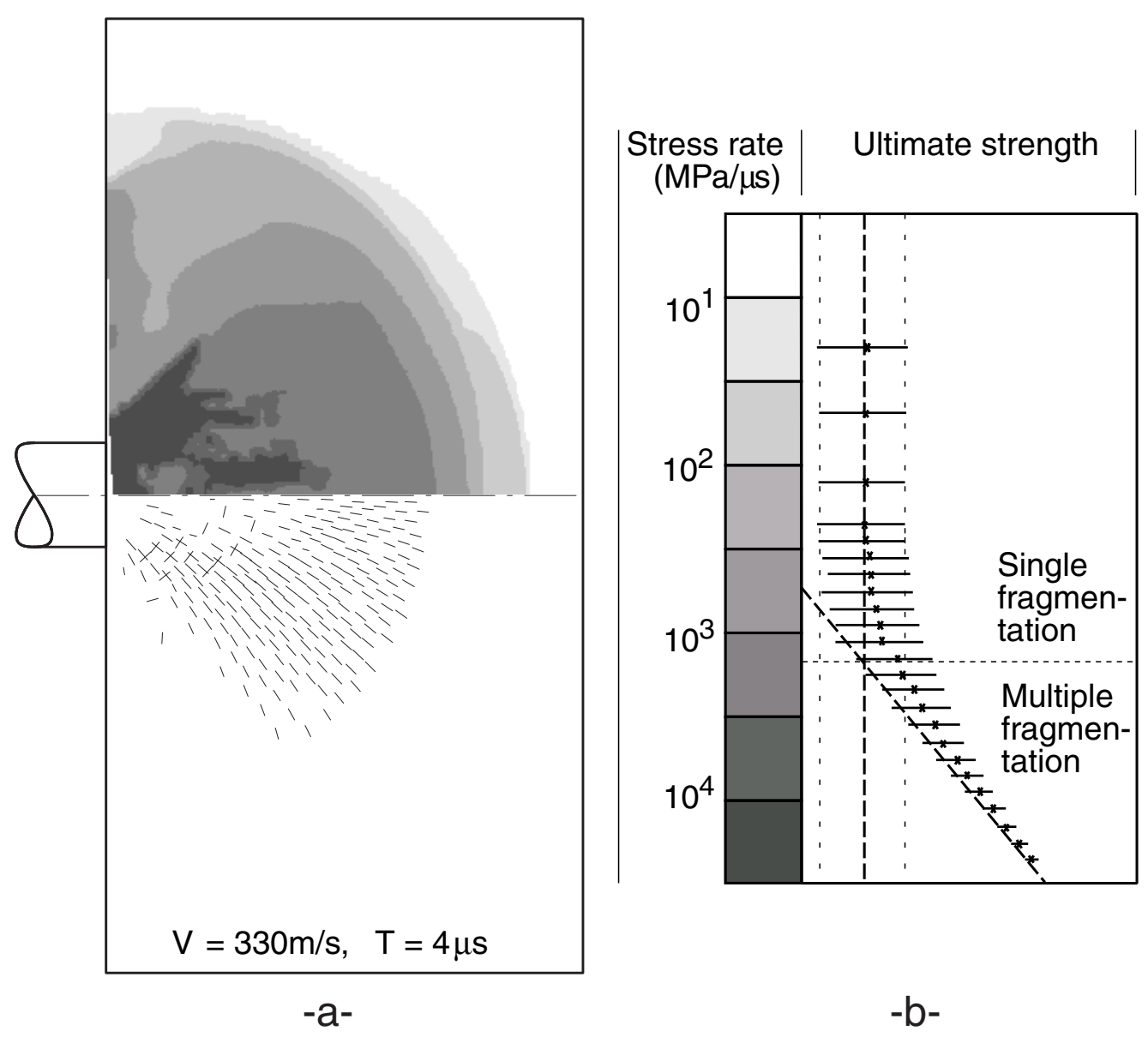

Figure 6: Denoual and Hild

Figure 6: Requirements to be fulfilled to use a continuum damage model can be checked by using a maximum stress rate map.

a- Example of maximum stress rate for the first eigen stress $4 \mu s$ after impact (top) and the corresponding damaged zone (bottom). The line density is a function of broken flaws density and the line direction is that of cracking.

b- The stress rate associated to locations where damage is generated leads to a complex behavior, neither discrete nor continuum. 

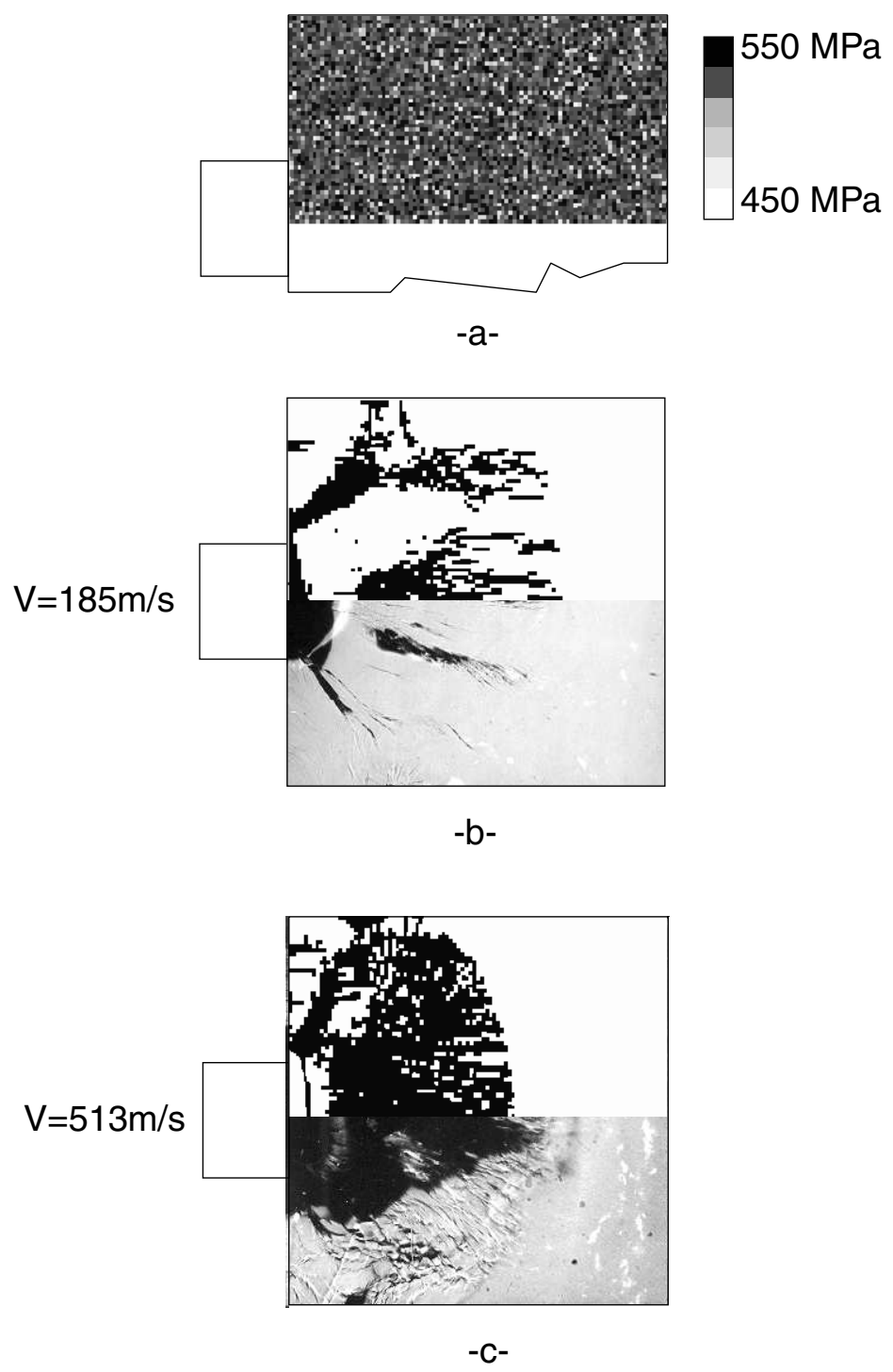

Figure 7: Denoual and Hild

Figure 7: Numerical simulations of a SiC-B ceramic in an EOI configuration $(50 \times 100 \times 10$ elements of $\left.1 \times 1 \times 1 \mathrm{~mm}^{3}\right)$.

a- Example of random failure stress $\sigma_{k}$ for the first defect able to break in each FE cell. b- and c- Tile upper part: simulations (multi-scale model), tile lower part: experiments. Damaged zones $\left(D_{1}>0.5\right.$ in the dark zones) for two impact velocities. 


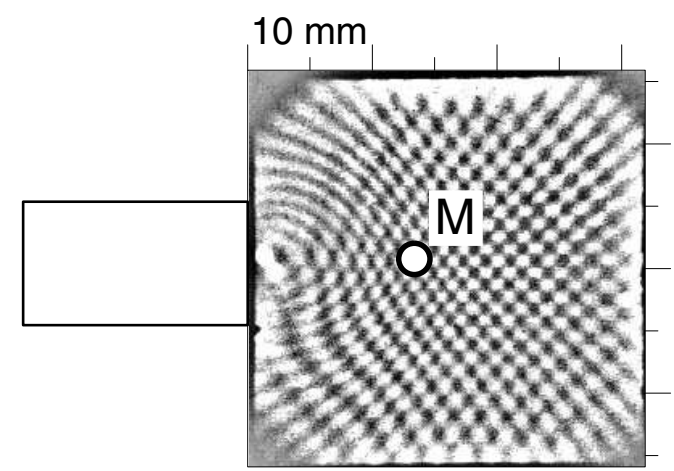

$-a-$

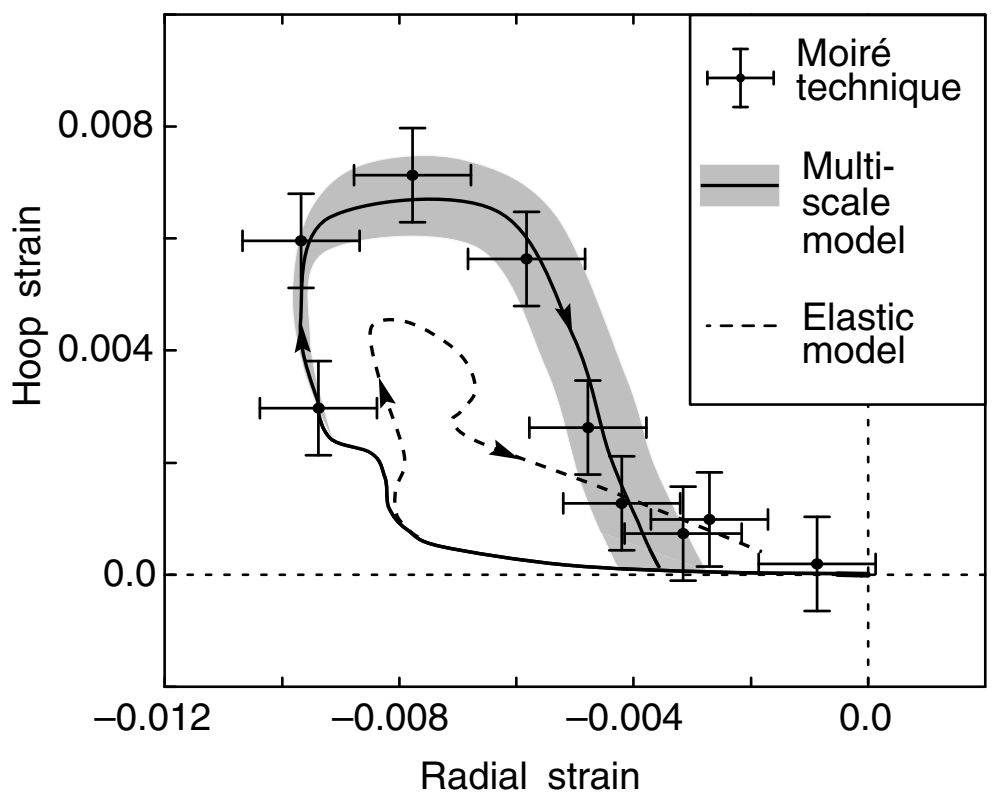

$-b-$

Figure 8: Denoual and Hild

Figure 8: a- Typical example of moiré fringes.

b- Strain change given by a moiré technique (dots) and by the multi-scale model (plain curve: average, grey bandwidth: \pm standard deviation). 


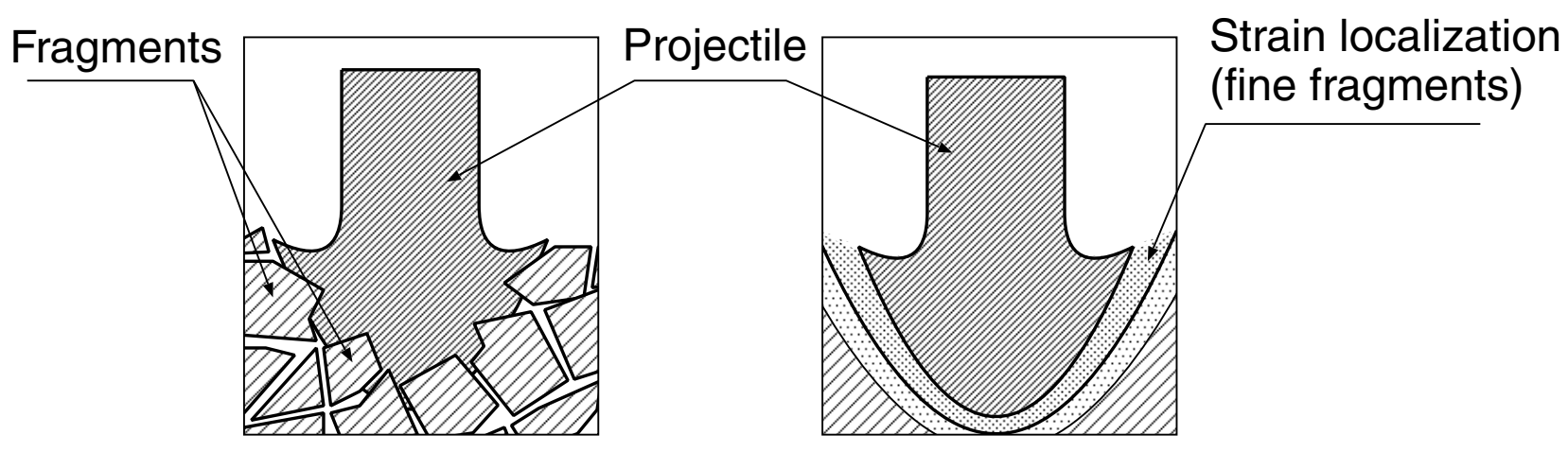

Figure 9: Denoual and Hild

Figure 9: Strains around a penetrating projectile when the fragmentation is coarse (left) and fine (right). 


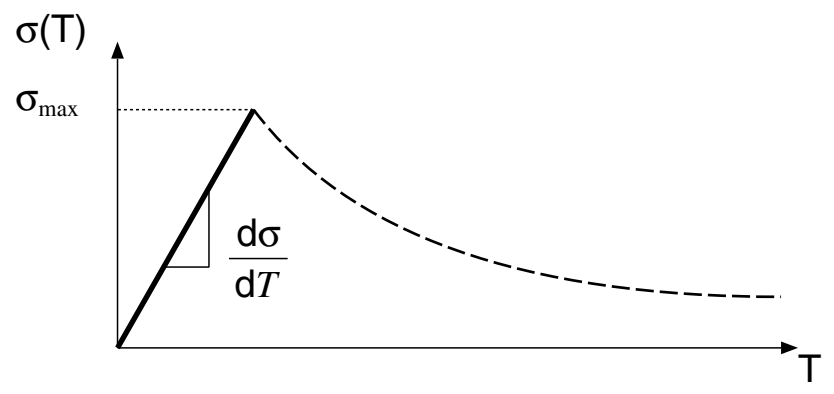

-a-

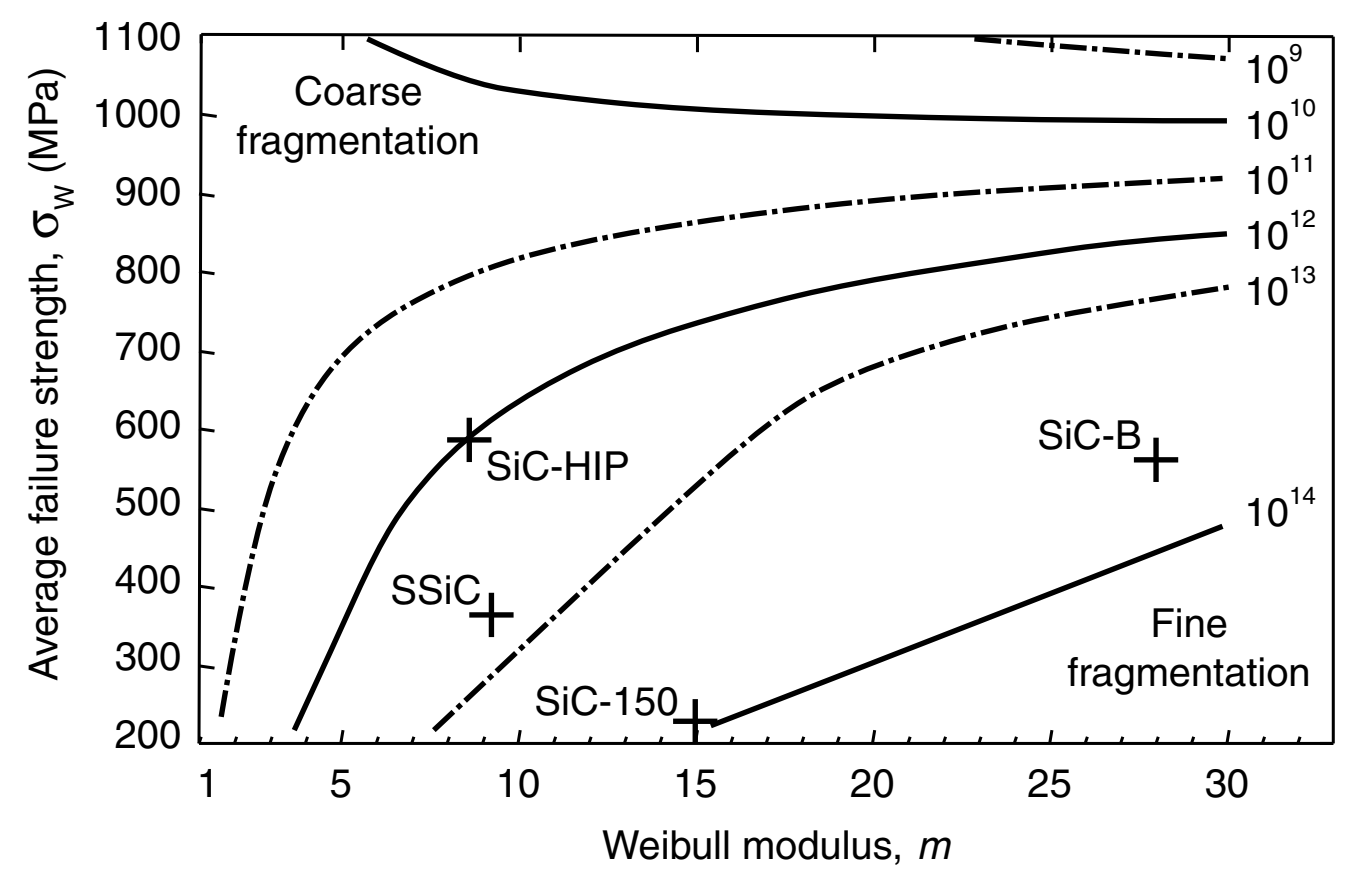

-b-

Figure 10: Denoual and Hild

Figure 10: a- Stress history for material optimization. The dashed curve is any positive monotonically decreasing function.

b- Broken flaw density map as function of Weibull modulus $m$ and average failure stress $\sigma_{\mathrm{w}}$. The four grades of silicon carbide are depicted by crosses. 